\title{
Supplement \\ More Stable Estimation of the STARTS Model: \\ A Bayesian Approach Using Markov Chain Monte Carlo Techniques
}

\section{Table of Contents}

Supplement A: Additional Results for Main Simulation Study 1

Supplement B: Additional Simulation for Large Sample Sizes $\quad 29$

Supplement C: Additional Simulation on Impact of Misspecified Prior Distributions 32

Supplement D: Additional Simulation on Highest Posterior Density (HPD) Intervals 35

Supplement E: Additional Simulation on Small Sample Sizes and Many Measurement 36 Points

Supplement F: Additional Simulation on More Extreme Conditions 37

Supplement G: Software Code for the Data Analysis Example 44 
Enclosed in this document are the supplemental materials for our article entitled "More Stable Estimation of the STARTS Model: A Bayesian Approach Using Markov Chain Monte Carlo Techniques".

Supplement A contains additional tables and figures to the results reported in the article. Supplement B contains the results of an additional simulation study in which we also looked at very large sample sizes. Supplement $\mathrm{C}$ contains the results of an additional simulation study in which we investigated the impact of a misspecified prior distribution. Supplement $\mathrm{D}$ contains the results of an additional simulation study in which we compared the coverage rates produced highest posterior density (HPD) interval estimates and the BCI. Supplement E contains the results of an additional simulation study in which we investigated the performance of the Bayesian approach with small sample sizes and a larger number of measurement points. Supplement F contains the results of an additional simulation study in which more extreme conditions were studied. Supplement G contains the computer code that was used to calculate the data analysis example.

\section{Supplement A: Additional Results for Main Simulation Study}

The following pages contain the additional results for the main simulation study. Table A1 shows the percentage of estimation problems for the ML approach with unconstrained estimation. The relative bias, relative RMSE, and the coverage rates for the estimators of the STARTS model parameters as a function of the ratio of the variance of the stable trait variance to the autoregressive trait, the size of the state variance, and the magnitude of the stability coefficient are presented in Tables A2 to A20. The relative bias, relative RMSE, and the coverage rates as a function of sample size and number of measurement waves are presented in Figures A1 to A7. 
Table A1

Percentage of Solutions with Estimation Problems (no Convergence, Inadmissible Parameter Estimates or Singular Information Matrix) for Unconstrained Maximum Likelihood Estimation of the STARTS Model by Sample Size (N), Number of Measurement Waves (W), and STARTS Model Parameters

\begin{tabular}{|c|c|c|c|c|c|c|c|c|c|c|c|}
\hline \multirow[b]{3}{*}{$\beta$} & \multirow[b]{3}{*}{$\sigma_{S}^{2}$} & \multirow[b]{3}{*}{ Ratio } & \multicolumn{3}{|c|}{$W=4$} & \multicolumn{3}{|c|}{$W=6$} & \multicolumn{3}{|c|}{$W=9$} \\
\hline & & & \multicolumn{3}{|c|}{ Sample size $(N)$} & \multicolumn{3}{|c|}{ Sample size $(N)$} & \multicolumn{3}{|c|}{ Sample size $(N)$} \\
\hline & & & 200 & 500 & 1000 & 200 & 500 & 1000 & 200 & 500 & 1000 \\
\hline 0.2 & 0.1 & $3: 1$ & 58.4 & 53.8 & 47.8 & 45.8 & 39.9 & 36.5 & 41.3 & 35.1 & 24.7 \\
\hline 0.2 & 0.1 & $1: 1$ & 66.0 & 53.5 & 51.0 & 48.1 & 44.6 & 40.0 & 41.9 & 38.8 & 31.4 \\
\hline 0.2 & 0.1 & $1: 3$ & 74.1 & 59.2 & 53.8 & 49.9 & 43.5 & 41.6 & 45.4 & 42.4 & 39.9 \\
\hline 0.2 & 0.3 & $3: 1$ & 59.4 & 50.1 & 46.4 & 47.3 & 39.9 & 35.4 & 39.7 & 34.7 & 25.2 \\
\hline 0.2 & 0.3 & $1: 1$ & 71.2 & 63.2 & 52.6 & 51.0 & 41.7 & 32.8 & 39.5 & 30.0 & 23.8 \\
\hline 0.2 & 0.3 & $1: 3$ & 76.9 & 63.7 & 52.4 & 51.2 & 38.9 & 31.8 & 37.5 & 33.5 & 23.6 \\
\hline 0.5 & 0.1 & $3: 1$ & 54.8 & 30.4 & 16.8 & 16.3 & 4.5 & 1.0 & 3.8 & 0.5 & 0.0 \\
\hline 0.5 & 0.1 & $1: 1$ & 50.8 & 33.1 & 20.0 & 19.4 & 10.7 & 3.3 & 9.2 & 2.0 & 0.0 \\
\hline 0.5 & 0.1 & 1:3 & 61.4 & 46.0 & 26.5 & 28.0 & 14.3 & 5.1 & 15.4 & 4.7 & 0.6 \\
\hline 0.5 & 0.3 & $3: 1$ & 62.4 & 51.4 & 36.7 & 31.8 & 11.0 & 2.6 & 6.7 & 0.6 & 0.0 \\
\hline 0.5 & 0.3 & $1: 1$ & 62.4 & 43.0 & 25.4 & 24.8 & 3.4 & 0.6 & 3.3 & 0.0 & 0.0 \\
\hline 0.5 & 0.3 & $1: 3$ & 67.6 & 47.7 & 29.7 & 22.7 & 5.9 & 0.9 & 2.0 & 0.1 & 0.0 \\
\hline 0.8 & 0.1 & $3: 1$ & 52.8 & 35.7 & 25.2 & 14.5 & 6.5 & 0.9 & 0.2 & 0.0 & 0.0 \\
\hline 0.8 & 0.1 & $1: 1$ & 50.5 & 33.0 & 25.0 & 16.7 & 4.7 & 1.7 & 1.5 & 0.0 & 0.0 \\
\hline 0.8 & 0.1 & $1: 3$ & 53.5 & 42.7 & 33.9 & 29.7 & 17.5 & 7.9 & 11.0 & 1.6 & 0.5 \\
\hline 0.8 & 0.3 & $3: 1$ & 60.3 & 51.4 & 45.8 & 38.4 & 23.7 & 15.8 & 15.7 & 5.1 & 0.8 \\
\hline 0.8 & 0.3 & $1: 1$ & 61.4 & 51.3 & 38.9 & 34.8 & 22.0 & 10.4 & 8.8 & 1.0 & 0.1 \\
\hline 0.8 & 0.3 & $1: 3$ & 65.3 & 50.1 & 43.7 & 38.3 & 29.4 & 18.2 & 18.9 & 7.5 & 1.0 \\
\hline
\end{tabular}

Note. $\beta=$ population stability parameter. $\sigma_{S}^{2}=$ state variance. Ratio $=$ ratio of stable trait variance to autoregressive trait variance. Values larger than $25 \%$ are printed in bold. 
Table A2

Relative Bias, Relative RMSE, and Coverage for the Maximum Likelihood and the Bayesian Approach (with Different Priors) as a Function of the Ratio of Stable Trait to Autoregressive Trait Variance for a Small Sample Size $(N=200)$, Four Measurement Waves $(W=4)$, a Large State Variance $\left(\sigma_{S}^{2}=.3\right)$ and a Medium Sized Stability Coefficient $(\beta=.5)$

\begin{tabular}{|c|c|c|c|c|c|c|c|c|c|c|c|c|c|}
\hline & \multirow[b]{2}{*}{ Ratio } & \multicolumn{4}{|c|}{ Relative bias } & \multicolumn{4}{|c|}{ Relative RMSE } & \multicolumn{4}{|c|}{ Coverage } \\
\hline & & ML & $v_{0}=1$ & $v_{0}=3$ & $v_{0}=10$ & ML & $v_{0}=1$ & $v_{0}=3$ & $v_{0}=10$ & ML & $v_{0}=1$ & $v_{0}=3$ & $v_{0}=10$ \\
\hline \multirow{3}{*}{$\hat{\sigma}_{T}^{2}$} & $3: 1$ & -0.34 & -0.02 & -0.05 & -0.10 & 0.59 & 0.14 & 0.14 & 0.16 & 98.1 & 97.1 & 95.7 & 92.8 \\
\hline & $1: 1$ & -0.29 & 0.00 & -0.02 & -0.03 & 0.60 & 0.21 & 0.18 & 0.15 & 97.3 & 99.1 & 99.1 & 99.1 \\
\hline & $1: 3$ & -0.23 & 0.10 & 0.19 & 0.34 & 0.72 & 0.38 & 0.34 & 0.39 & 95.9 & 100 & 99.6 & 90.8 \\
\hline \multirow{3}{*}{$\hat{\sigma}_{A}^{2}$} & $3: 1$ & 1.47 & 0.18 & 0.24 & 0.35 & 1.82 & 0.51 & 0.41 & 0.41 & 98.6 & 96.0 & 95.6 & 89.0 \\
\hline & $1: 1$ & 0.53 & 0.08 & 0.02 & -0.04 & 0.65 & 0.34 & 0.27 & 0.18 & 99.8 & 97.0 & 98.4 & 99.7 \\
\hline & $1: 3$ & 0.24 & 0.02 & -0.08 & -0.19 & 0.35 & 0.25 & 0.23 & 0.25 & 99.1 & 98.2 & 97.8 & 92.2 \\
\hline \multirow{3}{*}{$\hat{\sigma}_{S}^{2}$} & $3: 1$ & -0.29 & 0.04 & -0.02 & -0.09 & 0.60 & 0.15 & 0.12 & 0.13 & 89.4 & 99.6 & 99.3 & 97.1 \\
\hline & $1: 1$ & -0.28 & 0.10 & 0.07 & 0.05 & 0.64 & 0.27 & 0.20 & 0.14 & 91.7 & 99.9 & 100 & 100 \\
\hline & $1: 3$ & -0.27 & 0.01 & 0.07 & 0.15 & 0.66 & 0.38 & 0.28 & 0.23 & 95.7 & 99.6 & 99.8 & 99.8 \\
\hline \multirow{3}{*}{$\hat{\beta}$} & $3: 1$ & 0.12 & -0.05 & -0.01 & 0.04 & 0.72 & 0.44 & 0.17 & 0.07 & 83.9 & 100 & 100 & 100 \\
\hline & $1: 1$ & 0.03 & -0.01 & 0.01 & 0.01 & 0.57 & 0.31 & 0.13 & 0.06 & 95.9 & 100 & 100 & 100 \\
\hline & $1: 3$ & -0.02 & -0.10 & -0.08 & -0.06 & 0.43 & 0.20 & 0.12 & 0.08 & 99.3 & 100 & 100 & 100 \\
\hline
\end{tabular}

Note. RMSE $=$ root mean square error. $\mathrm{ML}=$ Maximum Likelihood with constrained estimation. $v_{0}$ $=$ prior sample size selected the for the prior distributions in the Bayesian approach. Ratio = ratio of stable trait variance to autoregressive trait variance. Relative biases smaller than -.10 or larger than .10 and coverage rates smaller than $91 \%$ and larger than $98 \%$ are written in bold. 
Table A3

Relative Bias, Relative RMSE, and Coverage for the Maximum Likelihood and the Bayesian Approach (with Different Priors) as a Function of the Ratio of Stable Trait to Autoregressive Trait Variance for a Large Sample Size $(N=1000)$, Four Measurement Waves $(W=4)$, a Large State Variance $\left(\sigma_{S}^{2}=.3\right)$ and a Medium Sized Stability Coefficient $(\beta=.5)$

\begin{tabular}{|c|c|c|c|c|c|c|c|c|c|c|c|c|c|}
\hline & \multirow[b]{2}{*}{ Ratio } & \multicolumn{4}{|c|}{ Relative bias } & \multicolumn{4}{|c|}{ Relative RMSE } & \multicolumn{4}{|c|}{ Coverage } \\
\hline & & ML & $v_{0}=1$ & $v_{0}=3$ & $v_{0}=10$ & ML & $v_{0}=1$ & $v_{0}=3$ & $v_{0}=10$ & ML & $v_{0}=1$ & $v_{0}=3$ & $v_{0}=10$ \\
\hline \multirow{3}{*}{$\hat{\sigma}_{T}^{2}$} & $3: 1$ & -0.22 & 0.01 & -0.01 & -0.03 & 0.46 & 0.07 & 0.07 & 0.07 & 96.4 & 98.5 & 98.3 & 96.6 \\
\hline & $1: 1$ & -0.16 & 0.03 & 0.02 & 0.00 & 0.42 & 0.13 & 0.11 & 0.09 & 94.2 & 98.5 & 98.9 & 98.7 \\
\hline & $1: 3$ & -0.15 & 0.08 & 0.11 & 0.17 & 0.55 & 0.26 & 0.22 & 0.22 & 94.5 & 99.7 & 99.7 & 96.9 \\
\hline \multirow{3}{*}{$\hat{\sigma}_{A}^{2}$} & $3: 1$ & 0.99 & 0.11 & 0.12 & 0.17 & 1.38 & 0.28 & 0.24 & 0.23 & 99.8 & 94.4 & 94.5 & 90.8 \\
\hline & $1: 1$ & 0.29 & 0.08 & 0.05 & 0.01 & 0.43 & 0.19 & 0.16 & 0.13 & 99.1 & 94.4 & 96.1 & 96.9 \\
\hline & $1: 3$ & 0.14 & 0.05 & 0.01 & -0.05 & 0.23 & 0.14 & 0.12 & 0.12 & 99.3 & 95.7 & 97.0 & 96.3 \\
\hline \multirow{3}{*}{$\hat{\sigma}_{S}^{2}$} & $3: 1$ & -0.22 & 0.06 & 0.03 & -0.03 & 0.49 & 0.14 & 0.09 & 0.07 & 86.1 & 99.7 & 99.6 & 98.8 \\
\hline & $1: 1$ & -0.15 & 0.08 & 0.06 & 0.04 & 0.46 & 0.21 & 0.15 & 0.10 & 92.1 & 99.7 & 99.9 & 99.9 \\
\hline & $1: 3$ & -0.16 & -0.01 & -0.02 & 0.02 & 0.48 & 0.25 & 0.18 & 0.13 & 94.7 & 99.4 & 99.4 & 99.7 \\
\hline \multirow{3}{*}{$\hat{\beta}$} & $3: 1$ & 0.04 & -0.02 & -0.04 & 0.00 & 0.61 & 0.48 & 0.25 & 0.09 & 78.5 & 100 & 100 & 100 \\
\hline & $1: 1$ & 0.03 & 0.01 & 0.00 & 0.02 & 0.44 & 0.35 & 0.19 & 0.08 & 92.7 & 100 & 100 & 100 \\
\hline & $1: 3$ & -0.01 & -0.06 & -0.07 & -0.07 & 0.33 & 0.23 & 0.14 & 0.08 & 97.6 & 100 & 100 & 100 \\
\hline
\end{tabular}

Note. RMSE $=$ root mean square error. $\mathrm{ML}=$ Maximum Likelihood with constrained estimation. $v_{0}$ $=$ prior sample size selected the for the prior distributions in the Bayesian approach. Ratio $=$ ratio of stable trait variance to autoregressive trait variance. Relative biases smaller than -.10 or larger than .10 and coverage rates smaller than $91 \%$ and larger than $98 \%$ are written in bold. 
Table A4

Relative Bias, Relative RMSE, and Coverage for the Maximum Likelihood and the Bayesian Approach (with Different Priors) as a Function of the Ratio of Stable Trait to Autoregressive Trait Variance for a Small Sample Size $(N=200)$, Nine Measurement Waves $(W=9)$, a Large State Variance $\left(\sigma_{S}^{2}=.3\right)$ and a Medium Sized Stability Coefficient $(\beta=.5)$

\begin{tabular}{|c|c|c|c|c|c|c|c|c|c|c|c|c|c|}
\hline & \multirow[b]{2}{*}{ Ratio } & \multicolumn{4}{|c|}{ Relative bias } & \multicolumn{4}{|c|}{ Relative RMSE } & \multicolumn{4}{|c|}{ Coverage } \\
\hline & & ML & $v_{0}=1$ & $v_{0}=3$ & $v_{0}=10$ & ML & $v_{0}=1$ & $v_{0}=3$ & $v_{0}=10$ & ML & $v_{0}=1$ & $v_{0}=3$ & $v_{0}=10$ \\
\hline \multirow{3}{*}{$\hat{\sigma}_{T}^{2}$} & $3: 1$ & -0.03 & -0.03 & -0.03 & -0.04 & 0.17 & 0.12 & 0.11 & 0.11 & 96.2 & 95.3 & 94.9 & 95.1 \\
\hline & $1: 1$ & -0.02 & -0.03 & -0.03 & -0.03 & 0.18 & 0.16 & 0.15 & 0.14 & 94.3 & 94.1 & 94.6 & 95.4 \\
\hline & $1: 3$ & -0.05 & -0.03 & 0.02 & 0.12 & 0.27 & 0.23 & 0.20 & 0.20 & 96.0 & 96.3 & 97.1 & 94.9 \\
\hline \multirow{3}{*}{$\hat{\sigma}_{A}^{2}$} & $3: 1$ & 0.27 & 0.06 & 0.08 & 0.14 & 0.59 & 0.25 & 0.21 & 0.20 & 98.7 & 96.9 & 97.2 & 93.8 \\
\hline & $1: 1$ & 0.09 & 0.04 & 0.02 & -0.01 & 0.25 & 0.18 & 0.15 & 0.12 & 97.8 & 97.2 & 98.0 & 99.0 \\
\hline & $1: 3$ & 0.04 & 0.03 & 0.00 & -0.05 & 0.18 & 0.15 & 0.13 & 0.12 & 96.6 & 96.6 & 97.6 & 97.6 \\
\hline \multirow{3}{*}{$\hat{\sigma}_{S}^{2}$} & $3: 1$ & -0.11 & 0.02 & -0.01 & -0.05 & 0.33 & 0.15 & 0.12 & 0.09 & 93.0 & 98.9 & 99.4 & 98.7 \\
\hline & $1: 1$ & -0.08 & 0.01 & 0.02 & 0.03 & 0.32 & 0.23 & 0.17 & 0.11 & 94.7 & 97.5 & 98.9 & 99.8 \\
\hline & $1: 3$ & -0.05 & 0.00 & 0.02 & 0.05 & 0.34 & 0.27 & 0.21 & 0.15 & 94.9 & 98.4 & 99.0 & 99.3 \\
\hline \multirow{3}{*}{$\hat{\beta}$} & $3: 1$ & 0.00 & -0.05 & -0.05 & -0.06 & 0.39 & 0.34 & 0.23 & 0.13 & 91.0 & 98.7 & 100 & 100 \\
\hline & 1:1 & 0.00 & -0.02 & 0.00 & 0.01 & 0.26 & 0.24 & 0.17 & 0.10 & 94.8 & 97.8 & 99.3 & 99.9 \\
\hline & $1: 3$ & 0.01 & -0.01 & -0.01 & -0.01 & 0.21 & 0.18 & 0.13 & 0.08 & 96.0 & 99.2 & 99.9 & 100 \\
\hline
\end{tabular}

Note. RMSE $=$ root mean square error. $\mathrm{ML}=$ Maximum Likelihood with constrained estimation. $v_{0}$ $=$ prior sample size selected the for the prior distributions in the Bayesian approach. Ratio = ratio of stable trait variance to autoregressive trait variance. Relative biases smaller than -.10 or larger than .10 and coverage rates smaller than $91 \%$ and larger than $98 \%$ are written in bold. 
Table A5

Relative Bias, Relative RMSE, and Coverage for the Maximum Likelihood and the Bayesian Approach (with Different Priors) as a Function of the Ratio of Stable Trait to Autoregressive Trait Variance for a Large Sample Size $(N=1000)$, Nine Measurement Waves $(W=9)$, a Large State Variance $\left(\sigma_{S}^{2}=.3\right)$ and a Medium Sized Stability Coefficient $(\beta=.5)$

\begin{tabular}{|c|c|c|c|c|c|c|c|c|c|c|c|c|c|}
\hline & \multirow[b]{2}{*}{ Ratio } & \multicolumn{4}{|c|}{ Relative bias } & \multicolumn{4}{|c|}{ Relative RMSE } & \multicolumn{4}{|c|}{ Coverage } \\
\hline & & ML & $v_{0}=1$ & $v_{0}=3$ & $v_{0}=10$ & ML & $v_{0}=1$ & $v_{0}=3$ & $v_{0}=10$ & ML & $v_{0}=1$ & $v_{0}=3$ & $v_{0}=10$ \\
\hline \multirow{3}{*}{$\hat{\sigma}_{T}^{2}$} & $3: 1$ & -0.01 & -0.01 & -0.01 & -0.01 & 0.05 & 0.05 & 0.05 & 0.05 & 94.9 & 94.7 & 94.5 & 94.9 \\
\hline & $1: 1$ & 0.00 & -0.01 & -0.01 & -0.01 & 0.07 & 0.07 & 0.07 & 0.07 & 93.5 & 93.3 & 93.8 & 93.9 \\
\hline & $1: 3$ & -0.01 & -0.01 & 0.00 & 0.03 & 0.12 & 0.11 & 0.11 & 0.10 & 95.8 & 95.3 & 96.4 & 96.2 \\
\hline \multirow{3}{*}{$\hat{\sigma}_{A}^{2}$} & $3: 1$ & 0.04 & 0.02 & 0.03 & 0.05 & 0.16 & 0.14 & 0.13 & 0.11 & 96.9 & 95.3 & 95.5 & 95.4 \\
\hline & $1: 1$ & 0.02 & 0.01 & 0.01 & 0.00 & 0.10 & 0.09 & 0.09 & 0.08 & 95.9 & 94.9 & 95.5 & 96.5 \\
\hline & $1: 3$ & 0.01 & 0.01 & 0.01 & 0.00 & 0.08 & 0.08 & 0.07 & 0.07 & 94.5 & 94.0 & 94.8 & 96.5 \\
\hline \multirow{3}{*}{$\hat{\sigma}_{S}^{2}$} & $3: 1$ & -0.02 & 0.00 & 0.00 & -0.02 & 0.11 & 0.10 & 0.09 & 0.07 & 93.4 & 94.4 & 96.0 & 96.9 \\
\hline & $1: 1$ & -0.02 & -0.01 & 0.00 & 0.01 & 0.13 & 0.12 & 0.11 & 0.09 & 96.2 & 94.7 & 95.5 & 97.3 \\
\hline & $1: 3$ & -0.01 & 0.00 & 0.00 & 0.01 & 0.15 & 0.15 & 0.13 & 0.11 & 94.6 & 94.7 & 95.9 & 96.8 \\
\hline \multirow{3}{*}{$\hat{\beta}$} & $3: 1$ & 0.00 & -0.01 & -0.02 & -0.04 & 0.18 & 0.18 & 0.16 & 0.12 & 95.1 & 95.1 & 95.7 & 98.4 \\
\hline & $1: 1$ & 0.00 & -0.01 & 0.00 & 0.00 & 0.12 & 0.13 & 0.11 & 0.09 & 94.4 & 94.5 & 95.4 & 97.5 \\
\hline & $1: 3$ & 0.00 & 0.00 & 0.00 & -0.01 & 0.10 & 0.10 & 0.09 & 0.07 & 93.8 & 94.2 & 96.0 & 98.1 \\
\hline
\end{tabular}

Note. RMSE $=$ root mean square error. $\mathrm{ML}=$ Maximum Likelihood with constrained estimation. $v_{0}$ $=$ prior sample size selected the for the prior distributions in the Bayesian approach. Ratio $=$ ratio of stable trait variance to autoregressive trait variance. Relative biases smaller than -.10 or larger than .10 and coverage rates smaller than $91 \%$ and larger than $98 \%$ are written in bold. 
Table A6

Relative Bias, Relative RMSE, and Coverage for the Maximum Likelihood and the Bayesian Approach (with Different Priors) as a Function of the Ratio of Stable Trait to Autoregressive Trait Variance for a Small Sample Size $(N=200)$, Four Measurement Waves $(W=4)$, a Large State Variance $\left(\sigma_{S}^{2}=.3\right)$ and a Small Sized Stability Coefficient $(\beta=.2)$

\begin{tabular}{|c|c|c|c|c|c|c|c|c|c|c|c|c|c|}
\hline & \multirow[b]{2}{*}{ Ratio } & \multicolumn{4}{|c|}{ Relative bias } & \multicolumn{4}{|c|}{ Relative RMSE } & \multicolumn{4}{|c|}{ Coverage } \\
\hline & & ML & $v_{0}=1$ & $v_{0}=3$ & $v_{0}=10$ & ML & $v_{0}=1$ & $v_{0}=3$ & $v_{0}=10$ & ML & $v_{0}=1$ & $v_{0}=3$ & $v_{0}=10$ \\
\hline \multirow{3}{*}{$\hat{\boldsymbol{\sigma}}_{T}^{2}$} & $3: 1$ & -0.29 & -0.07 & -0.10 & -0.15 & 0.50 & 0.15 & 0.16 & 0.19 & 97.9 & 94.1 & 91.4 & 83.4 \\
\hline & $1: 1$ & -0.26 & -0.09 & -0.13 & -0.16 & 0.51 & 0.19 & 0.20 & 0.20 & 97.8 & 94.7 & 94.1 & 92.8 \\
\hline & $1: 3$ & -0.31 & -0.12 & -0.07 & 0.05 & 0.60 & 0.28 & 0.20 & 0.15 & 98.7 & 99.0 & 99.9 & 99.9 \\
\hline \multirow{3}{*}{$\hat{\sigma}_{A}^{2}$} & $3: 1$ & 1.01 & -0.12 & 0.05 & 0.27 & 1.54 & 0.42 & 0.30 & 0.33 & 89.8 & 99.4 & 99.4 & 96.9 \\
\hline & $1: 1$ & 0.36 & -0.33 & -0.28 & -0.21 & 0.71 & 0.46 & 0.36 & 0.25 & 92.3 & 100 & 99.6 & 99.0 \\
\hline & $1: 3$ & 0.11 & -0.46 & -0.47 & -0.46 & 0.50 & 0.55 & 0.51 & 0.47 & 96.6 & 98.5 & 91.2 & 57.3 \\
\hline \multirow{3}{*}{$\hat{\sigma}_{S}^{2}$} & $3: 1$ & -0.10 & 0.26 & 0.17 & 0.07 & 0.66 & 0.29 & 0.21 & 0.12 & 57.0 & 100 & 100 & 100 \\
\hline & $1: 1$ & -0.12 & 0.61 & 0.52 & 0.42 & 0.86 & 0.65 & 0.55 & 0.44 & 69.0 & 100 & 98.1 & 83.9 \\
\hline & $1: 3$ & -0.01 & 0.89 & 0.87 & 0.80 & 0.99 & 1.00 & 0.91 & 0.82 & 77.7 & 99.1 & 91.5 & 36.5 \\
\hline \multirow{3}{*}{$\hat{\beta}$} & $3: 1$ & 2.03 & 0.95 & 1.22 & 1.47 & 2.85 & 1.54 & 1.31 & 1.48 & 75.1 & 98.5 & 82.4 & 0.0 \\
\hline & 1:1 & 1.24 & 0.82 & 1.17 & 1.33 & 2.17 & 1.20 & 1.22 & 1.33 & 90.3 & 97.5 & 80.9 & 0.0 \\
\hline & $1: 3$ & 0.90 & 0.51 & 0.84 & 1.08 & 1.68 & 0.70 & 0.88 & 1.09 & 97.7 & 98.4 & 92.4 & 0.0 \\
\hline
\end{tabular}

Note. RMSE $=$ root mean square error. $\mathrm{ML}=$ Maximum Likelihood with constrained estimation. $v_{0}$ $=$ prior sample size selected the for the prior distributions in the Bayesian approach. Ratio = ratio of stable trait variance to autoregressive trait variance. Relative biases smaller than -.10 or larger than .10 and coverage rates smaller than $91 \%$ and larger than $98 \%$ are written in bold. 
Table A7

Relative Bias, Relative RMSE, and Coverage for the Maximum Likelihood and the Bayesian Approach (with Different Priors) as a Function of the Ratio of Stable Trait to Autoregressive Trait Variance for a Large Sample Size $(N=1000)$, Four Measurement Waves $(W=4)$, a Large State Variance $\left(\sigma_{S}^{2}=.3\right)$ and a Small Sized Stability Coefficient $(\beta=.2)$

\begin{tabular}{|c|c|c|c|c|c|c|c|c|c|c|c|c|c|}
\hline & \multirow[b]{2}{*}{ Ratio } & \multicolumn{4}{|c|}{ Relative bias } & \multicolumn{4}{|c|}{ Relative RMSE } & \multicolumn{4}{|c|}{ Coverage } \\
\hline & & ML & $v_{0}=1$ & $v_{0}=3$ & $v_{0}=10$ & ML & $v_{0}=1$ & $v_{0}=3$ & $v_{0}=10$ & ML & $v_{0}=1$ & $v_{0}=3$ & $v_{0}=10$ \\
\hline \multirow{3}{*}{$\hat{\boldsymbol{\sigma}}_{T}^{2}$} & $3: 1$ & -0.20 & -0.03 & -0.04 & -0.07 & 0.39 & 0.07 & 0.08 & 0.10 & 98.2 & 92.9 & 90.3 & 80.6 \\
\hline & $1: 1$ & -0.17 & -0.04 & -0.07 & -0.10 & 0.38 & 0.09 & 0.11 & 0.13 & 98.3 & 93.7 & 91.5 & 84.0 \\
\hline & $1: 3$ & -0.21 & -0.08 & -0.09 & -0.06 & 0.44 & 0.18 & 0.16 & 0.12 & 98.1 & 94.4 & 95.7 & 98.4 \\
\hline \multirow{3}{*}{$\hat{\sigma}_{A}^{2}$} & $3: 1$ & 0.92 & -0.27 & -0.17 & -0.03 & 1.32 & 0.35 & 0.24 & 0.13 & 100 & 99.8 & 99.9 & 99.7 \\
\hline & $1: 1$ & 0.31 & -0.36 & -0.35 & -0.30 & 0.61 & 0.41 & 0.38 & 0.33 & 95.7 & 99.0 & 91.9 & 65.4 \\
\hline & $1: 3$ & 0.10 & -0.37 & -0.40 & -0.43 & 0.44 & 0.41 & 0.42 & 0.44 & 92.9 & 95.3 & 84.4 & 35.8 \\
\hline \multirow{3}{*}{$\hat{\sigma}_{S}^{2}$} & $3: 1$ & -0.20 & 0.32 & 0.26 & 0.18 & 0.67 & 0.33 & 0.27 & 0.19 & 66.0 & 99.2 & 100 & 98.5 \\
\hline & $1: 1$ & -0.16 & 0.63 & 0.59 & 0.52 & 0.79 & 0.66 & 0.61 & 0.53 & 77.9 & 95.7 & 85.1 & 28.5 \\
\hline & $1: 3$ & -0.03 & 0.81 & 0.82 & 0.82 & 0.89 & 0.88 & 0.85 & 0.83 & 78.5 & 95.3 & 83.7 & 20.0 \\
\hline \multirow{3}{*}{$\hat{\beta}$} & $3: 1$ & 1.34 & 0.78 & 0.90 & 1.24 & 2.35 & 1.55 & 1.15 & 1.27 & 83.9 & 99.1 & 94.2 & 4.4 \\
\hline & $1: 1$ & 0.76 & 0.81 & 1.04 & 1.26 & 1.67 & 1.36 & 1.16 & 1.27 & 91.2 & 97.0 & 82.9 & 0.4 \\
\hline & $1: 3$ & 0.63 & 0.53 & 0.78 & 0.97 & 1.35 & 0.86 & 0.86 & 0.98 & 96.6 & 98.9 & 89.1 & 0.8 \\
\hline
\end{tabular}

Note. RMSE $=$ root mean square error. $\mathrm{ML}=$ Maximum Likelihood with constrained estimation. $v_{0}$ $=$ prior sample size selected the for the prior distributions in the Bayesian approach. Ratio $=$ ratio of stable trait variance to autoregressive trait variance. Relative biases smaller than -.10 or larger than .10 and coverage rates smaller than $91 \%$ and larger than $98 \%$ are written in bold. 
Table A8

Relative Bias, Relative RMSE, and Coverage for the Maximum Likelihood and the Bayesian Approach (with Different Priors) as a Function of the Ratio of Stable Trait to Autoregressive Trait Variance for a Small Sample Size $(N=200)$, Nine Measurement Waves $(W=9)$, a Large State Variance $\left(\sigma_{S}^{2}=.3\right)$ and a Small Sized Stability Coefficient $(\beta=.2)$

\begin{tabular}{|c|c|c|c|c|c|c|c|c|c|c|c|c|c|}
\hline & \multirow[b]{2}{*}{ Ratio } & \multicolumn{4}{|c|}{ Relative bias } & \multicolumn{4}{|c|}{ Relative RMSE } & \multicolumn{4}{|c|}{ Coverage } \\
\hline & & ML & $v_{0}=1$ & $v_{0}=3$ & $v_{0}=10$ & ML & $v_{0}=1$ & $v_{0}=3$ & $v_{0}=10$ & ML & $v_{0}=1$ & $v_{0}=3$ & $v_{0}=10$ \\
\hline \multirow{3}{*}{$\hat{\boldsymbol{\sigma}}_{T}^{2}$} & $3: 1$ & -0.09 & -0.03 & -0.04 & -0.06 & 0.28 & 0.12 & 0.12 & 0.12 & 94.3 & 95.2 & 94.2 & 93.4 \\
\hline & $1: 1$ & -0.05 & -0.04 & -0.04 & -0.05 & 0.22 & 0.13 & 0.13 & 0.13 & 95.4 & 95.9 & 95.4 & 95.2 \\
\hline & $1: 3$ & -0.06 & -0.06 & -0.04 & 0.02 & 0.23 & 0.18 & 0.16 & 0.14 & 95.1 & 95.6 & 96.8 & 97.5 \\
\hline \multirow{3}{*}{$\hat{\sigma}_{A}^{2}$} & $3: 1$ & 0.63 & -0.38 & -0.26 & -0.10 & 1.21 & 0.44 & 0.30 & 0.14 & 87.3 & 100 & 100 & 100 \\
\hline & $1: 1$ & 0.15 & -0.44 & -0.43 & -0.37 & 0.62 & 0.50 & 0.45 & 0.38 & 82.8 & 97.8 & 93.4 & 66.8 \\
\hline & $1: 3$ & 0.06 & -0.43 & -0.46 & -0.46 & 0.47 & 0.49 & 0.48 & 0.47 & 83.2 & 95.6 & 85.7 & 33.7 \\
\hline \multirow{3}{*}{$\hat{\sigma}_{S}^{2}$} & $3: 1$ & -0.24 & 0.26 & 0.19 & 0.12 & 0.67 & 0.31 & 0.22 & 0.14 & 75.2 & 99.3 & 99.4 & 99.8 \\
\hline & $1: 1$ & -0.12 & 0.57 & 0.55 & 0.49 & 0.77 & 0.64 & 0.58 & 0.50 & 77.9 & 97.0 & 93.2 & 50.0 \\
\hline & $1: 3$ & -0.07 & 0.79 & 0.84 & 0.83 & 0.86 & 0.90 & 0.87 & 0.85 & 82.0 & 95.7 & 86.2 & 21.1 \\
\hline \multirow{3}{*}{$\hat{\beta}$} & $3: 1$ & 0.75 & 0.18 & 0.40 & 0.76 & 1.73 & 0.86 & 0.60 & 0.79 & 90.1 & 99.4 & 98.3 & 40.0 \\
\hline & $1: 1$ & 0.53 & 0.42 & 0.67 & 0.96 & 1.30 & 0.89 & 0.80 & 0.99 & 92.7 & 97.3 & 89.3 & 8.2 \\
\hline & $1: 3$ & 0.38 & 0.40 & 0.68 & 0.95 & 1.03 & 0.78 & 0.80 & 0.97 & 94.7 & 96.5 & 85.6 & 4.6 \\
\hline
\end{tabular}

Note. RMSE $=$ root mean square error. $\mathrm{ML}=$ Maximum Likelihood with constrained estimation. $v_{0}$ $=$ prior sample size selected the for the prior distributions in the Bayesian approach. Ratio = ratio of stable trait variance to autoregressive trait variance. Relative biases smaller than -.10 or larger than .10 and coverage rates smaller than $91 \%$ and larger than $98 \%$ are written in bold. 
Table A9

Relative Bias, Relative RMSE, and Coverage for the Maximum Likelihood and the Bayesian Approach (with Different Priors) as a Function of the Ratio of Stable Trait to Autoregressive Trait Variance for a Large Sample Size $(N=1000)$, Nine Measurement Waves $(W=9)$, a Large State Variance $\left(\sigma_{S}^{2}=.3\right)$ and a Small Sized Stability Coefficient $(\beta=.2)$

\begin{tabular}{|c|c|c|c|c|c|c|c|c|c|c|c|c|c|}
\hline & \multirow[b]{2}{*}{ Ratio } & \multicolumn{4}{|c|}{ Relative bias } & \multicolumn{4}{|c|}{ Relative RMSE } & \multicolumn{4}{|c|}{ Coverage } \\
\hline & & ML & $v_{0}=1$ & $v_{0}=3$ & $v_{0}=10$ & ML & $v_{0}=1$ & $v_{0}=3$ & $v_{0}=10$ & ML & $v_{0}=1$ & $v_{0}=3$ & $v_{0}=10$ \\
\hline \multirow{3}{*}{$\hat{\boldsymbol{\sigma}}_{T}^{2}$} & $3: 1$ & 0.00 & -0.01 & -0.01 & -0.01 & 0.06 & 0.05 & 0.05 & 0.05 & 94.7 & 94.6 & 94.7 & 93.7 \\
\hline & $1: 1$ & 0.00 & -0.01 & -0.01 & -0.02 & 0.06 & 0.06 & 0.06 & 0.06 & 94.1 & 94.3 & 93.8 & 93.6 \\
\hline & $1: 3$ & -0.01 & -0.02 & -0.02 & -0.02 & 0.08 & 0.08 & 0.08 & 0.08 & 93.7 & 93.7 & 93.9 & 95.1 \\
\hline \multirow{3}{*}{$\hat{\sigma}_{A}^{2}$} & $3: 1$ & 0.37 & -0.29 & -0.28 & -0.21 & 0.94 & 0.42 & 0.33 & 0.23 & 84.9 & 97.3 & 98.5 & 99.1 \\
\hline & $1: 1$ & 0.14 & -0.23 & -0.33 & -0.38 & 0.52 & 0.38 & 0.37 & 0.39 & 86.1 & 94.6 & 89.7 & 48.9 \\
\hline & $1: 3$ & 0.05 & -0.19 & -0.30 & -0.39 & 0.38 & 0.33 & 0.35 & 0.40 & 86.5 & 94.5 & 86.9 & 36.9 \\
\hline \multirow{3}{*}{$\hat{\sigma}_{S}^{2}$} & $3: 1$ & -0.21 & 0.18 & 0.18 & 0.14 & 0.57 & 0.26 & 0.20 & 0.15 & 85.2 & 96.1 & 98.2 & 98.6 \\
\hline & $1: 1$ & -0.16 & 0.28 & 0.39 & 0.46 & 0.62 & 0.45 & 0.44 & 0.47 & 86.7 & 94.7 & 89.3 & 44.6 \\
\hline & $1: 3$ & -0.09 & 0.33 & 0.54 & 0.71 & 0.67 & 0.59 & 0.62 & 0.72 & 86.4 & 94.2 & 86.7 & 35.1 \\
\hline \multirow{3}{*}{$\hat{\beta}$} & $3: 1$ & 0.19 & 0.09 & 0.23 & 0.43 & 0.84 & 0.61 & 0.46 & 0.47 & 94.3 & 97.0 & 97.1 & 83.8 \\
\hline & $1: 1$ & 0.12 & 0.14 & 0.38 & 0.67 & 0.58 & 0.51 & 0.54 & 0.71 & 96.0 & 94.8 & 90.9 & 31.4 \\
\hline & $1: 3$ & 0.13 & 0.15 & 0.37 & 0.68 & 0.50 & 0.46 & 0.52 & 0.72 & 95.8 & 94.2 & 86.3 & 25.1 \\
\hline
\end{tabular}

Note. RMSE $=$ root mean square error. $\mathrm{ML}=$ Maximum Likelihood with constrained estimation. $v_{0}$ $=$ prior sample size selected the for the prior distributions in the Bayesian approach. Ratio $=$ ratio of stable trait variance to autoregressive trait variance. Relative biases smaller than -.10 or larger than .10 and coverage rates smaller than $91 \%$ and larger than $98 \%$ are written in bold. 
Table A10

Relative Bias, Relative RMSE, and Coverage for the Maximum Likelihood and the Bayesian Approach (with Different Priors) as a Function of the Ratio of Stable Trait to Autoregressive Trait Variance for a Small Sample Size $(N=200)$, Four Measurement Waves $(W=4)$, a Large State Variance $\left(\sigma_{S}^{2}=.3\right)$ and a Large Sized Stability Coefficient $(\beta=.8)$

\begin{tabular}{|c|c|c|c|c|c|c|c|c|c|c|c|c|c|}
\hline & \multirow[b]{2}{*}{ Ratio } & \multicolumn{4}{|c|}{ Relative bias } & \multicolumn{4}{|c|}{ Relative RMSE } & \multicolumn{4}{|c|}{ Coverage } \\
\hline & & ML & $v_{0}=1$ & $v_{0}=3$ & $v_{0}=10$ & ML & $v_{0}=1$ & $v_{0}=3$ & $v_{0}=10$ & ML & $v_{0}=1$ & $v_{0}=3$ & $v_{0}=10$ \\
\hline \multirow{3}{*}{$\hat{\boldsymbol{\sigma}}_{T}^{2}$} & $3: 1$ & -0.33 & 0.11 & 0.08 & 0.03 & 0.65 & 0.18 & 0.16 & 0.14 & 93.5 & 99.8 & 98.7 & 97.4 \\
\hline & $1: 1$ & -0.21 & 0.40 & 0.37 & 0.32 & 0.75 & 0.46 & 0.42 & 0.37 & 81.3 & 99.2 & 92.0 & 75.7 \\
\hline & $1: 3$ & 0.02 & 1.19 & 1.19 & 1.19 & 1.16 & 1.29 & 1.26 & 1.24 & 79.3 & 96.8 & 61.7 & 3.4 \\
\hline \multirow{3}{*}{$\hat{\sigma}_{A}^{2}$} & $3: 1$ & 1.45 & -0.09 & 0.01 & 0.15 & 1.95 & 0.36 & 0.25 & 0.22 & 98.3 & 99.3 & 99.4 & 98.9 \\
\hline & $1: 1$ & 0.44 & -0.23 & -0.24 & -0.24 & 0.69 & 0.35 & 0.31 & 0.27 & 99.5 & 99.3 & 95.9 & 90.5 \\
\hline & 1:3 & 0.12 & -0.26 & -0.31 & -0.36 & 0.31 & 0.33 & 0.35 & 0.38 & 98.8 & 92.9 & 78.3 & 44.2 \\
\hline \multirow{3}{*}{$\hat{\sigma}_{S}^{2}$} & $3: 1$ & -0.29 & -0.08 & -0.14 & -0.21 & 0.53 & 0.13 & 0.17 & 0.22 & 94.8 & 94.4 & 84.1 & 51.1 \\
\hline & $1: 1$ & -0.27 & -0.08 & -0.13 & -0.17 & 0.50 & 0.18 & 0.18 & 0.19 & 97.4 & 94.1 & 92.9 & 84.7 \\
\hline & $1: 3$ & -0.21 & -0.11 & -0.14 & -0.14 & 0.45 & 0.22 & 0.20 & 0.18 & 97.6 & 94.4 & 94.5 & 94.7 \\
\hline \multirow{3}{*}{$\hat{\beta}$} & $3: 1$ & -0.18 & -0.36 & -0.36 & -0.33 & 0.49 & 0.48 & 0.38 & 0.33 & 82.5 & 100 & 99.4 & 0.0 \\
\hline & $1: 1$ & -0.20 & -0.28 & -0.32 & -0.32 & 0.43 & 0.37 & 0.33 & 0.32 & 90.1 & 99.7 & 91.9 & 0.0 \\
\hline & $1: 3$ & -0.15 & -0.23 & -0.29 & -0.32 & 0.34 & 0.29 & 0.30 & 0.32 & 93.5 & 91.0 & 56.9 & 0.0 \\
\hline
\end{tabular}

Note. RMSE $=$ root mean square error. $\mathrm{ML}=$ Maximum Likelihood with constrained estimation. $v_{0}$ $=$ prior sample size selected the for the prior distributions in the Bayesian approach. Ratio = ratio of stable trait variance to autoregressive trait variance. Relative biases smaller than -.10 or larger than .10 and coverage rates smaller than $91 \%$ and larger than $98 \%$ are written in bold. 
Table A11

Relative Bias, Relative RMSE, and Coverage for the Maximum Likelihood and the Bayesian Approach (with Different Priors) as a Function of the Ratio of Stable Trait to Autoregressive Trait Variance for a Large Sample Size $(N=1000)$, Four Measurement Waves $(W=4)$, a Large State Variance $\left(\sigma_{S}^{2}=.3\right)$ and a Large Sized Stability Coefficient $(\beta=.8)$

\begin{tabular}{|c|c|c|c|c|c|c|c|c|c|c|c|c|c|}
\hline & \multirow[b]{2}{*}{ Ratio } & \multicolumn{4}{|c|}{ Relative bias } & \multicolumn{4}{|c|}{ Relative RMSE } & \multicolumn{4}{|c|}{ Coverage } \\
\hline & & ML & $v_{0}=1$ & $v_{0}=3$ & $v_{0}=10$ & ML & $v_{0}=1$ & $v_{0}=3$ & $v_{0}=10$ & ML & $v_{0}=1$ & $v_{0}=3$ & $v_{0}=10$ \\
\hline \multirow{3}{*}{$\hat{\sigma}_{T}^{2}$} & $3: 1$ & -0.34 & 0.14 & 0.13 & 0.11 & 0.64 & 0.16 & 0.15 & 0.13 & 82.1 & 98.4 & 95.5 & 86.1 \\
\hline & $1: 1$ & -0.21 & 0.41 & 0.41 & 0.40 & 0.69 & 0.43 & 0.42 & 0.41 & 78.6 & 96.4 & 86.3 & 28.6 \\
\hline & $1: 3$ & 0.00 & 1.04 & 1.12 & 1.18 & 0.99 & 1.12 & 1.16 & 1.20 & 82.4 & 93.0 & 63.3 & 0.2 \\
\hline \multirow{3}{*}{$\hat{\sigma}_{A}^{2}$} & $3: 1$ & 1.22 & -0.20 & -0.16 & -0.06 & 1.83 & 0.27 & 0.22 & 0.13 & 99.2 & 100 & 99.9 & 99.9 \\
\hline & $1: 1$ & 0.30 & -0.24 & -0.25 & -0.26 & 0.63 & 0.27 & 0.28 & 0.28 & 91.2 & 99.7 & 91.9 & 51.1 \\
\hline & $1: 3$ & 0.05 & -0.24 & -0.27 & -0.31 & 0.28 & 0.27 & 0.29 & 0.32 & 86.9 & 94.6 & 68.2 & 13.9 \\
\hline \multirow{3}{*}{$\hat{\sigma}_{S}^{2}$} & $3: 1$ & -0.14 & -0.03 & -0.07 & -0.13 & 0.34 & 0.07 & 0.09 & 0.14 & 97.0 & 94.5 & 86.8 & 45.1 \\
\hline & $1: 1$ & -0.10 & -0.05 & -0.08 & -0.13 & 0.25 & 0.10 & 0.11 & 0.14 & 98.7 & 92.8 & 88.7 & 70.3 \\
\hline & $1: 3$ & -0.08 & -0.07 & -0.10 & -0.14 & 0.22 & 0.13 & 0.14 & 0.16 & 99.0 & 90.8 & 85.9 & 73.0 \\
\hline \multirow{3}{*}{$\hat{\beta}$} & $3: 1$ & -0.13 & -0.25 & -0.32 & -0.35 & 0.40 & 0.42 & 0.38 & 0.35 & 90.1 & 98.9 & 94.5 & 6.8 \\
\hline & $1: 1$ & -0.11 & -0.17 & -0.26 & -0.31 & 0.29 & 0.29 & 0.29 & 0.31 & 95.1 & 96.6 & 86.5 & 0.3 \\
\hline & $1: 3$ & -0.09 & -0.13 & -0.21 & -0.28 & 0.22 & 0.22 & 0.24 & 0.28 & 94.4 & 91.9 & 60.4 & 0.0 \\
\hline
\end{tabular}

Note. RMSE $=$ root mean square error. $\mathrm{ML}=$ Maximum Likelihood with constrained estimation. $v_{0}$ $=$ prior sample size selected the for the prior distributions in the Bayesian approach. Ratio $=$ ratio of stable trait variance to autoregressive trait variance. Relative biases smaller than -.10 or larger than .10 and coverage rates smaller than $91 \%$ and larger than $98 \%$ are written in bold. 
Table A12

Relative Bias, Relative RMSE, and Coverage for the Maximum Likelihood and the Bayesian Approach (with Different Priors) as a Function of the Ratio of Stable Trait to Autoregressive Trait Variance for a Small Sample Size $(N=200)$, Nine Measurement Waves $(W=9)$, a Large State Variance $\left(\sigma_{S}^{2}=.3\right)$ and a Large Sized Stability Coefficient $(\beta=.8)$

\begin{tabular}{|c|c|c|c|c|c|c|c|c|c|c|c|c|c|}
\hline & \multirow[b]{2}{*}{ Ratio } & \multicolumn{4}{|c|}{ Relative bias } & \multicolumn{4}{|c|}{ Relative RMSE } & \multicolumn{4}{|c|}{ Coverage } \\
\hline & & ML & $v_{0}=1$ & $v_{0}=3$ & $v_{0}=10$ & ML & $v_{0}=1$ & $v_{0}=3$ & $v_{0}=10$ & ML & $v_{0}=1$ & $v_{0}=3$ & $v_{0}=10$ \\
\hline \multirow{3}{*}{$\hat{\sigma}_{T}^{2}$} & $3: 1$ & -0.18 & 0.02 & 0.04 & 0.04 & 0.43 & 0.14 & 0.13 & 0.13 & 99.1 & 97.6 & 97.1 & 94.5 \\
\hline & $1: 1$ & -0.15 & 0.05 & 0.12 & 0.18 & 0.43 & 0.24 & 0.22 & 0.24 & 97.2 & 97.1 & 95.3 & 83.9 \\
\hline & $1: 3$ & -0.15 & 0.19 & 0.40 & 0.63 & 0.63 & 0.45 & 0.51 & 0.68 & 94.9 & 96.6 & 91.6 & 35.3 \\
\hline \multirow{3}{*}{$\hat{\sigma}_{A}^{2}$} & $3: 1$ & 0.57 & -0.04 & -0.04 & 0.01 & 1.19 & 0.19 & 0.16 & 0.12 & 98.2 & 98.7 & 99.1 & 99.0 \\
\hline & $1: 1$ & 0.15 & -0.05 & -0.09 & -0.13 & 0.40 & 0.17 & 0.16 & 0.17 & 94.9 & 98.4 & 97.4 & 87.1 \\
\hline & $1: 3$ & 0.05 & -0.07 & -0.12 & -0.17 & 0.20 & 0.14 & 0.16 & 0.20 & 93.9 & 97.1 & 89.1 & 62.9 \\
\hline \multirow{3}{*}{$\hat{\sigma}_{S}^{2}$} & $3: 1$ & -0.02 & -0.03 & -0.06 & -0.13 & 0.11 & 0.08 & 0.09 & 0.14 & 97.0 & 94.8 & 90.5 & 61.7 \\
\hline & $1: 1$ & -0.02 & -0.03 & -0.06 & -0.11 & 0.11 & 0.10 & 0.11 & 0.13 & 95.5 & 93.7 & 89.9 & 77.0 \\
\hline & $1: 3$ & -0.01 & -0.04 & -0.06 & -0.11 & 0.11 & 0.10 & 0.11 & 0.14 & 96.6 & 94.5 & 91.8 & 79.8 \\
\hline \multirow{3}{*}{$\hat{\beta}$} & $3: 1$ & -0.01 & -0.06 & -0.15 & -0.27 & 0.18 & 0.19 & 0.21 & 0.28 & 84.6 & 95.2 & 88.7 & 26.9 \\
\hline & $1: 1$ & -0.01 & -0.03 & -0.09 & -0.18 & 0.12 & 0.12 & 0.13 & 0.19 & 92.3 & 94.9 & 88.9 & 35.6 \\
\hline & $1: 3$ & -0.01 & -0.03 & -0.08 & -0.15 & 0.09 & 0.09 & 0.10 & 0.16 & 97.4 & 94.8 & 83.7 & 19.7 \\
\hline
\end{tabular}

Note. RMSE $=$ root mean square error. $\mathrm{ML}=$ Maximum Likelihood with constrained estimation. $v_{0}$ $=$ prior sample size selected the for the prior distributions in the Bayesian approach. Ratio = ratio of stable trait variance to autoregressive trait variance. Relative biases smaller than -.10 or larger than .10 and coverage rates smaller than $91 \%$ and larger than $98 \%$ are written in bold. 
Table A13

Relative Bias, Relative RMSE, and Coverage for the Maximum Likelihood and the Bayesian Approach (with Different Priors) as a Function of the Ratio of Stable Trait to Autoregressive Trait Variance for a Large Sample Size $(N=1000)$, Nine Measurement Waves $(W=9)$, a Large State Variance $\left(\sigma_{S}^{2}=.3\right)$ and a Large Sized Stability Coefficient $(\beta=.8)$

\begin{tabular}{|c|c|c|c|c|c|c|c|c|c|c|c|c|c|}
\hline & \multirow[b]{2}{*}{ Ratio } & \multicolumn{4}{|c|}{ Relative bias } & \multicolumn{4}{|c|}{ Relative RMSE } & \multicolumn{4}{|c|}{ Coverage } \\
\hline & & ML & $v_{0}=1$ & $v_{0}=3$ & $v_{0}=10$ & ML & $v_{0}=1$ & $v_{0}=3$ & $v_{0}=10$ & ML & $v_{0}=1$ & $v_{0}=3$ & $v_{0}=10$ \\
\hline \multirow{3}{*}{$\hat{\sigma}_{T}^{2}$} & $3: 1$ & -0.04 & 0.00 & 0.02 & 0.04 & 0.15 & 0.08 & 0.07 & 0.07 & 98.0 & 96.2 & 96.2 & 92.6 \\
\hline & $1: 1$ & -0.03 & 0.01 & 0.04 & 0.11 & 0.19 & 0.15 & 0.13 & 0.15 & 95.9 & 94.4 & 94.6 & 87.4 \\
\hline & $1: 3$ & -0.03 & 0.06 & 0.18 & 0.36 & 0.34 & 0.28 & 0.28 & 0.39 & 95.4 & 96.9 & 93.8 & 63.2 \\
\hline \multirow{3}{*}{$\hat{\sigma}_{A}^{2}$} & $3: 1$ & 0.11 & -0.02 & -0.04 & -0.06 & 0.38 & 0.14 & 0.11 & 0.10 & 96.2 & 96.2 & 97.3 & 95.5 \\
\hline & $1: 1$ & 0.03 & -0.02 & -0.04 & -0.09 & 0.16 & 0.12 & 0.10 & 0.11 & 92.8 & 94.8 & 94.1 & 84.5 \\
\hline & $1: 3$ & 0.01 & -0.02 & -0.05 & -0.10 & 0.11 & 0.09 & 0.09 & 0.11 & 93.3 & 96.6 & 93.1 & 71.4 \\
\hline \multirow{3}{*}{$\hat{\sigma}_{S}^{2}$} & $3: 1$ & 0.00 & 0.00 & -0.01 & -0.04 & 0.04 & 0.04 & 0.04 & 0.05 & 95.7 & 96.0 & 94.9 & 80.5 \\
\hline & $1: 1$ & 0.00 & 0.00 & -0.01 & -0.04 & 0.04 & 0.04 & 0.04 & 0.05 & 94.0 & 94.7 & 94.6 & 86.3 \\
\hline & $1: 3$ & 0.00 & -0.01 & -0.02 & -0.04 & 0.05 & 0.04 & 0.05 & 0.06 & 95.1 & 95.5 & 93.5 & 86.4 \\
\hline \multirow{3}{*}{$\hat{\beta}$} & $3: 1$ & 0.00 & -0.01 & -0.03 & -0.10 & 0.08 & 0.08 & 0.08 & 0.12 & 94.5 & 95.8 & 94.6 & 73.4 \\
\hline & $1: 1$ & 0.00 & 0.00 & -0.02 & -0.06 & 0.06 & 0.06 & 0.06 & 0.08 & 93.4 & 93.7 & 93.5 & 76.5 \\
\hline & $1: 3$ & 0.00 & -0.01 & -0.03 & -0.06 & 0.04 & 0.04 & 0.04 & 0.07 & 95.4 & 96.2 & 92.5 & 57.5 \\
\hline
\end{tabular}

Note. RMSE $=$ root mean square error. $\mathrm{ML}=$ Maximum Likelihood with constrained estimation. $v_{0}$ $=$ prior sample size selected the for the prior distributions in the Bayesian approach. Ratio $=$ ratio of stable trait variance to autoregressive trait variance. Relative biases smaller than -.10 or larger than .10 and coverage rates smaller than $91 \%$ and larger than $98 \%$ are written in bold. 
Table A14

Relative Bias, Relative RMSE, and Coverage for the Maximum Likelihood and the Bayesian Approach (with Different Priors) as a Function of the Stability Parameter $\beta$ for a Small Sample Size $(N=$ 200), Four Measurement Waves $(W=4)$, a Large State Variance $\left(\sigma_{S}^{2}=.3\right)$ and Ratios of Stable Trait Variance to Autoregressive Variance of 1:1 or 3:1

\begin{tabular}{|c|c|c|c|c|c|c|c|c|c|c|c|c|c|}
\hline & \multirow[b]{2}{*}{$\beta$} & \multicolumn{4}{|c|}{ Relative bias } & \multicolumn{4}{|c|}{ Relative RMSE } & \multicolumn{4}{|c|}{ Coverage } \\
\hline & & ML & $v_{0}=1$ & $v_{0}=3$ & $v_{0}=10$ & ML & $v_{0}=1$ & $v_{0}=3$ & $v_{0}=10$ & ML & $v_{0}=1$ & $v_{0}=3$ & $v_{0}=10$ \\
\hline \multirow{4}{*}{$\hat{\sigma}_{T}^{2}$} & & Ratio o & f Stable & Trait to & Autoregr & essive & Trait I & ariance & of $1: 1$ & & & & \\
\hline & .2 & -0.26 & -0.09 & -0.13 & -0.16 & 0.51 & 0.19 & 0.20 & 0.20 & 97.8 & 94.7 & 94.1 & 92.8 \\
\hline & .5 & -0.29 & 0.00 & -0.02 & -0.03 & 0.60 & 0.21 & 0.18 & 0.15 & 97.3 & 99.1 & 99.1 & 99.1 \\
\hline & .8 & -0.21 & 0.40 & 0.37 & 0.32 & 0.75 & 0.46 & 0.42 & 0.37 & 81.3 & 99.2 & 92.0 & 75.7 \\
\hline \multirow{3}{*}{$\hat{\sigma}_{A}^{2}$} & .2 & 0.36 & -0.33 & -0.28 & -0.21 & 0.71 & 0.46 & 0.36 & 0.25 & 92.3 & 100 & 99.6 & 99.0 \\
\hline & .5 & 0.53 & 0.08 & 0.02 & -0.04 & 0.65 & 0.34 & 0.27 & 0.18 & 99.8 & 97.0 & 98.4 & 99.7 \\
\hline & .8 & 0.44 & -0.23 & -0.24 & -0.24 & 0.69 & 0.35 & 0.31 & 0.27 & 99.5 & 99.3 & 95.9 & 90.5 \\
\hline \multirow{3}{*}{$\hat{\sigma}_{S}^{2}$} & .2 & -0.12 & 0.61 & 0.52 & 0.42 & 0.86 & 0.65 & 0.55 & 0.44 & 69.0 & 100 & 98.1 & 83.9 \\
\hline & .5 & -0.28 & 0.10 & 0.07 & 0.05 & 0.64 & 0.27 & 0.20 & 0.14 & 91.7 & 99.9 & 100 & 100 \\
\hline & .8 & -0.27 & -0.08 & -0.13 & -0.17 & 0.50 & 0.18 & 0.18 & 0.19 & 97.4 & 94.1 & 92.9 & 84.7 \\
\hline \multirow{3}{*}{$\hat{\beta}$} & .2 & 1.24 & 0.82 & 1.17 & 1.33 & 2.17 & 1.20 & 1.22 & 1.33 & 90.3 & 97.5 & 80.9 & 0.0 \\
\hline & .5 & 0.03 & -0.01 & 0.01 & 0.01 & 0.57 & 0.31 & 0.13 & 0.06 & 95.9 & 100 & 100 & 100 \\
\hline & .8 & -0.20 & -0.28 & -0.32 & -0.32 & 0.43 & 0.37 & 0.33 & 0.32 & 90.1 & 99.7 & 91.9 & 0.0 \\
\hline \multirow{4}{*}{$\hat{\sigma}_{T}^{2}$} & & Ratio o & f Stable & Trait to & Autoregr & essive & Trait I & ariance & of $3: 1$ & & & & \\
\hline & .2 & -0.29 & -0.07 & -0.10 & -0.15 & 0.50 & 0.15 & 0.16 & 0.19 & 97.9 & 94.1 & 91.4 & 83.4 \\
\hline & .5 & -0.34 & -0.02 & -0.05 & -0.10 & 0.59 & 0.14 & 0.14 & 0.16 & 98.1 & 97.1 & 95.7 & 92.8 \\
\hline & .8 & -0.33 & 0.11 & 0.08 & 0.03 & 0.65 & 0.18 & 0.16 & 0.14 & 93.5 & 99.8 & 98.7 & 97.4 \\
\hline \multirow{3}{*}{$\hat{\sigma}_{A}^{2}$} & .2 & 1.01 & -0.12 & 0.05 & 0.27 & 1.54 & 0.42 & 0.30 & 0.33 & 89.8 & 99.4 & 99.4 & 96.9 \\
\hline & .5 & 1.47 & 0.18 & 0.24 & 0.35 & 1.82 & 0.51 & 0.41 & 0.41 & 98.6 & 96.0 & 95.6 & 89.0 \\
\hline & .8 & 1.45 & -0.09 & 0.01 & 0.15 & 1.95 & 0.36 & 0.25 & 0.22 & 98.3 & 99.3 & 99.4 & 98.9 \\
\hline \multirow{3}{*}{$\hat{\sigma}_{S}^{2}$} & .2 & -0.10 & 0.26 & 0.17 & 0.07 & 0.66 & 0.29 & 0.21 & 0.12 & 57.0 & 100 & 100 & 100 \\
\hline & .5 & -0.29 & 0.04 & -0.02 & -0.09 & 0.60 & 0.15 & 0.12 & 0.13 & 89.4 & 99.6 & 99.3 & 97.1 \\
\hline & .8 & -0.29 & -0.08 & -0.14 & -0.21 & 0.53 & 0.13 & 0.17 & 0.22 & 94.8 & 94.4 & 84.1 & 51.1 \\
\hline \multirow{3}{*}{$\hat{\beta}$} & .2 & 2.03 & 0.95 & 1.22 & 1.47 & 2.85 & 1.54 & 1.31 & 1.48 & 75.1 & 98.5 & 82.4 & 0.0 \\
\hline & .5 & 0.12 & -0.05 & -0.01 & 0.04 & 0.72 & 0.44 & 0.17 & 0.07 & 83.9 & 100 & 100 & 100 \\
\hline & .8 & -0.18 & -0.36 & -0.36 & -0.33 & 0.49 & 0.48 & 0.38 & 0.33 & 82.5 & 100 & 99.4 & 0.0 \\
\hline
\end{tabular}

Note. $\mathrm{RMSE}=$ root mean square error. $\mathrm{ML}=$ Maximum Likelihood with constrained estimation. $v_{0}$ $=$ prior sample size for prior distributions. $\beta=$ stability parameter. Relative biases smaller than -.10 or larger than .10 and coverage rates smaller than $91 \%$ and larger than $98 \%$ are written in bold. 
Table A15

Relative Bias, Relative RMSE, and Coverage for the Maximum Likelihood and the Bayesian Approach (with Different Priors) as a Function of the Stability Parameter $\beta$ for a Large Sample Size ( $N=$ 1000), Four Measurement Waves $(W=4)$, a Large State Variance $\left(\sigma_{S}^{2}=.3\right)$ and Ratios of Stable Trait Variance to Autoregressive Variance of 1:1 or 3:1

\begin{tabular}{|c|c|c|c|c|c|c|c|c|c|c|c|c|c|}
\hline & \multirow[b]{2}{*}{$\beta$} & \multicolumn{4}{|c|}{ Relative bias } & \multicolumn{4}{|c|}{ Relative RMSE } & \multicolumn{4}{|c|}{ Coverage } \\
\hline & & ML & $v_{0}=1$ & $v_{0}=3$ & $v_{0}=10$ & ML & $v_{0}=1$ & $v_{0}=3$ & $v_{0}=10$ & ML & $v_{0}=1$ & $v_{0}=3$ & $v_{0}=10$ \\
\hline \multirow{4}{*}{$\hat{\sigma}_{T}^{2}$} & & Ratio o & f Stable & Trait to & Autoregr & essive & Trait I & ariance & of $1: 1$ & & & & \\
\hline & .2 & -0.17 & -0.04 & -0.07 & -0.10 & 0.38 & 0.09 & 0.11 & 0.13 & 98.3 & 93.7 & 91.5 & 84.0 \\
\hline & .5 & -0.16 & 0.03 & 0.02 & 0.00 & 0.42 & 0.13 & 0.11 & 0.09 & 94.2 & 98.5 & 98.9 & 98.7 \\
\hline & .8 & -0.21 & 0.41 & 0.41 & 0.40 & 0.69 & 0.43 & 0.42 & 0.41 & 78.6 & 96.4 & 86.3 & 28.6 \\
\hline \multirow{3}{*}{$\hat{\sigma}_{A}^{2}$} & .2 & 0.31 & -0.36 & -0.35 & -0.30 & 0.61 & 0.41 & 0.38 & 0.33 & 95.7 & 99.0 & 91.9 & 65.4 \\
\hline & .5 & 0.29 & 0.08 & 0.05 & 0.01 & 0.43 & 0.19 & 0.16 & 0.13 & 99.1 & 94.4 & 96.1 & 96.9 \\
\hline & .8 & 0.30 & -0.24 & -0.25 & -0.26 & 0.63 & 0.27 & 0.28 & 0.28 & 91.2 & 99.7 & 91.9 & 51.1 \\
\hline \multirow{3}{*}{$\hat{\sigma}_{S}^{2}$} & .2 & -0.16 & 0.63 & 0.59 & 0.52 & 0.79 & 0.66 & 0.61 & 0.53 & 77.9 & 95.7 & 85.1 & 28.5 \\
\hline & .5 & -0.15 & 0.08 & 0.06 & 0.04 & 0.46 & 0.21 & 0.15 & 0.10 & 92.1 & 99.7 & 99.9 & 99.9 \\
\hline & .8 & -0.10 & -0.05 & -0.08 & -0.13 & 0.25 & 0.10 & 0.11 & 0.14 & 98.7 & 92.8 & 88.7 & 70.3 \\
\hline \multirow{3}{*}{$\hat{\beta}$} & .2 & 0.76 & 0.81 & 1.04 & 1.26 & 1.67 & 1.36 & 1.16 & 1.27 & 91.2 & 97.0 & 82.9 & 0.4 \\
\hline & .5 & 0.03 & 0.01 & 0.00 & 0.02 & 0.44 & 0.35 & 0.19 & 0.08 & 92.7 & 100 & 100 & 100 \\
\hline & .8 & -0.11 & -0.17 & -0.26 & -0.31 & 0.29 & 0.29 & 0.29 & 0.31 & 95.1 & 96.6 & 86.5 & 0.3 \\
\hline \multirow{4}{*}{$\hat{\sigma}_{T}^{2}$} & & Ratio o & f Stable & Trait to & Autoregr & essive & Trait I & ariance & of $3: 1$ & & & & \\
\hline & .2 & -0.20 & -0.03 & -0.04 & -0.07 & 0.39 & 0.07 & 0.08 & 0.10 & 98.2 & 92.9 & 90.3 & 80.6 \\
\hline & .5 & -0.22 & 0.01 & -0.01 & -0.03 & 0.46 & 0.07 & 0.07 & 0.07 & 96.4 & 98.5 & 98.3 & 96.6 \\
\hline & .8 & -0.34 & 0.14 & 0.13 & 0.11 & 0.64 & 0.16 & 0.15 & 0.13 & 82.1 & 98.4 & 95.5 & 86.1 \\
\hline \multirow{3}{*}{$\hat{\sigma}_{A}^{2}$} & .2 & 0.92 & -0.27 & -0.17 & -0.03 & 1.32 & 0.35 & 0.24 & 0.13 & 100 & 99.8 & 99.9 & 99.7 \\
\hline & .5 & 0.99 & 0.11 & 0.12 & 0.17 & 1.38 & 0.28 & 0.24 & 0.23 & 99.8 & 94.4 & 94.5 & 90.8 \\
\hline & .8 & 1.22 & -0.20 & -0.16 & -0.06 & 1.83 & 0.27 & 0.22 & 0.13 & 99.2 & 100 & 99.9 & 99.9 \\
\hline \multirow{3}{*}{$\hat{\sigma}_{S}^{2}$} & .2 & -0.20 & 0.32 & 0.26 & 0.18 & 0.67 & 0.33 & 0.27 & 0.19 & 66.0 & 99.2 & 100 & 98.5 \\
\hline & .5 & -0.22 & 0.06 & 0.03 & -0.03 & 0.49 & 0.14 & 0.09 & 0.07 & 86.1 & 99.7 & 99.6 & 98.8 \\
\hline & .8 & -0.14 & -0.03 & -0.07 & -0.13 & 0.34 & 0.07 & 0.09 & 0.14 & 97.0 & 94.5 & 86.8 & 45.1 \\
\hline \multirow{3}{*}{$\hat{\beta}$} & .2 & 1.34 & 0.78 & 0.90 & 1.24 & 2.35 & 1.55 & 1.15 & 1.27 & 83.9 & 99.1 & 94.2 & 4.4 \\
\hline & .5 & 0.04 & -0.02 & -0.04 & 0.00 & 0.61 & 0.48 & 0.25 & 0.09 & 78.5 & 100 & 100 & 100 \\
\hline & .8 & -0.13 & -0.25 & -0.32 & -0.35 & 0.40 & 0.42 & 0.38 & 0.35 & 90.1 & 98.9 & 94.5 & 6.8 \\
\hline
\end{tabular}

Note. RMSE $=$ root mean square error. $\mathrm{ML}=$ Maximum Likelihood with constrained estimation. $v_{0}$ $=$ prior sample size for prior distributions. $\beta=$ stability parameter. Relative biases smaller than -.10 or larger than .10 and coverage rates smaller than $91 \%$ and larger than $98 \%$ are written in bold. 
Table A16

Relative Bias, Relative RMSE, and Coverage for the Maximum Likelihood and the Bayesian Approach (with Different Priors) as a Function of the Stability Parameter $\beta$ for a Small Sample Size $(N=200)$, Nine Measurement Waves $(W=9)$, a Large State Variance $\left(\sigma_{S}^{2}=.3\right)$ and Ratios of Stable Trait Variance to Autoregressive Variance of 1:1 or 3:1

\begin{tabular}{|c|c|c|c|c|c|c|c|c|c|c|c|c|c|}
\hline & \multirow[b]{2}{*}{$\beta$} & \multicolumn{4}{|c|}{ Relative bias } & \multicolumn{4}{|c|}{ Relative RMSE } & \multicolumn{4}{|c|}{ Coverage } \\
\hline & & ML & $v_{0}=1$ & $v_{0}=3$ & $v_{0}=10$ & ML & $v_{0}=1$ & $v_{0}=3$ & $v_{0}=10$ & ML & $v_{0}=1$ & $v_{0}=3$ & $v_{0}=10$ \\
\hline \multirow{4}{*}{$\hat{\sigma}_{T}^{2}$} & & Ratio o & f Stable & Trait to & Autoregr & essive & Trait I & ariance & of $1: 1$ & & & & \\
\hline & .2 & -0.05 & -0.04 & -0.04 & -0.05 & 0.22 & 0.13 & 0.13 & 0.13 & 95.4 & 95.9 & 95.4 & 95.2 \\
\hline & .5 & -0.02 & -0.03 & -0.03 & -0.03 & 0.18 & 0.16 & 0.15 & 0.14 & 94.3 & 94.1 & 94.6 & 95.4 \\
\hline & .8 & -0.15 & 0.05 & 0.12 & 0.18 & 0.43 & 0.24 & 0.22 & 0.24 & 97.2 & 97.1 & 95.3 & 83.9 \\
\hline \multirow{3}{*}{$\hat{\sigma}_{A}^{2}$} & .2 & 0.15 & -0.44 & -0.43 & -0.37 & 0.62 & 0.50 & 0.45 & 0.38 & 82.8 & 97.8 & 93.4 & 66.8 \\
\hline & .5 & 0.09 & 0.04 & 0.02 & -0.01 & 0.25 & 0.18 & 0.15 & 0.12 & 97.8 & 97.2 & 98.0 & 99.0 \\
\hline & .8 & 0.15 & -0.05 & -0.09 & -0.13 & 0.40 & 0.17 & 0.16 & 0.17 & 94.9 & 98.4 & 97.4 & 87.1 \\
\hline \multirow{3}{*}{$\hat{\sigma}_{S}^{2}$} & .2 & -0.12 & 0.57 & 0.55 & 0.49 & 0.77 & 0.64 & 0.58 & 0.50 & 77.9 & 97.0 & 93.2 & 50.0 \\
\hline & .5 & -0.08 & 0.01 & 0.02 & 0.03 & 0.32 & 0.23 & 0.17 & 0.11 & 94.7 & 97.5 & 98.9 & 99.8 \\
\hline & .8 & -0.02 & -0.03 & -0.06 & -0.11 & 0.11 & 0.10 & 0.11 & 0.13 & 95.5 & 93.7 & 89.9 & 77.0 \\
\hline \multirow{3}{*}{$\hat{\beta}$} & .2 & 0.53 & 0.42 & 0.67 & 0.96 & 1.30 & 0.89 & 0.80 & 0.99 & 92.7 & 97.3 & 89.3 & 8.2 \\
\hline & .5 & 0.00 & -0.02 & 0.00 & 0.01 & 0.26 & 0.24 & 0.17 & 0.10 & 94.8 & 97.8 & 99.3 & 99.9 \\
\hline & .8 & -0.01 & -0.03 & -0.09 & -0.18 & 0.12 & 0.12 & 0.13 & 0.19 & 92.3 & 94.9 & 88.9 & 35.6 \\
\hline \multirow{4}{*}{$\hat{\sigma}_{T}^{2}$} & & Ratio o & f Stable & Trait to & Autoregr & essive & Trait I & ariance & of $3: 1$ & & & & \\
\hline & .2 & -0.09 & -0.03 & -0.04 & -0.06 & 0.28 & 0.12 & 0.12 & 0.12 & 94.3 & 95.2 & 94.2 & 93.4 \\
\hline & .5 & -0.03 & -0.03 & -0.03 & -0.04 & 0.17 & 0.12 & 0.11 & 0.11 & 96.2 & 95.3 & 94.9 & 95.1 \\
\hline & .8 & -0.18 & 0.02 & 0.04 & 0.04 & 0.43 & 0.14 & 0.13 & 0.13 & 99.1 & 97.6 & 97.1 & 94.5 \\
\hline \multirow{3}{*}{$\hat{\sigma}_{A}^{2}$} & .2 & 0.63 & -0.38 & -0.26 & -0.10 & 1.21 & 0.44 & 0.30 & 0.14 & 87.3 & 100 & 100 & 100 \\
\hline & .5 & 0.27 & 0.06 & 0.08 & 0.14 & 0.59 & 0.25 & 0.21 & 0.20 & 98.7 & 96.9 & 97.2 & 93.8 \\
\hline & .8 & 0.57 & -0.04 & -0.04 & 0.01 & 1.19 & 0.19 & 0.16 & 0.12 & 98.2 & 98.7 & 99.1 & 99.0 \\
\hline \multirow{3}{*}{$\hat{\sigma}_{S}^{2}$} & .2 & -0.24 & 0.26 & 0.19 & 0.12 & 0.67 & 0.31 & 0.22 & 0.14 & 75.2 & 99.3 & 99.4 & 99.8 \\
\hline & .5 & -0.11 & 0.02 & -0.01 & -0.05 & 0.33 & 0.15 & 0.12 & 0.09 & 93.0 & 98.9 & 99.4 & 98.7 \\
\hline & .8 & -0.02 & -0.03 & -0.06 & -0.13 & 0.11 & 0.08 & 0.09 & 0.14 & 97.0 & 94.8 & 90.5 & 61.7 \\
\hline \multirow{3}{*}{$\hat{\beta}$} & .2 & 0.75 & 0.18 & 0.40 & 0.76 & 1.73 & 0.86 & 0.60 & 0.79 & 90.1 & 99.4 & 98.3 & 40.0 \\
\hline & .5 & 0.00 & -0.05 & -0.05 & -0.06 & 0.39 & 0.34 & 0.23 & 0.13 & 91.0 & 98.7 & 100 & 100 \\
\hline & .8 & -0.01 & -0.06 & -0.15 & -0.27 & 0.18 & 0.19 & 0.21 & 0.28 & 84.6 & 95.2 & 88.7 & 26.9 \\
\hline
\end{tabular}

Note. RMSE $=$ root mean square error. $\mathrm{ML}=$ Maximum Likelihood with constrained estimation. $v_{0}$ $=$ prior sample size for prior distributions. $\beta=$ stability parameter. Relative biases smaller than -.10 or larger than .10 and coverage rates smaller than $91 \%$ and larger than $98 \%$ are written in bold. 
Table A17

Relative Bias, Relative RMSE, and Coverage for the Maximum Likelihood and the Bayesian Approach (with Different Priors) as a Function of the Stability Parameter $\beta$ for a Nine Sample Size ( $N=$ 1000), Nine Measurement Waves $(W=9)$, a Large State Variance $\left(\sigma_{S}^{2}=.3\right)$ and Ratios of Stable Trait Variance to Autoregressive Variance of 1:1 or 3:1

\begin{tabular}{|c|c|c|c|c|c|c|c|c|c|c|c|c|c|}
\hline & \multirow[b]{2}{*}{$\beta$} & \multicolumn{4}{|c|}{ Relative bias } & \multicolumn{4}{|c|}{ Relative RMSE } & \multicolumn{4}{|c|}{ Coverage } \\
\hline & & ML & $v_{0}=1$ & $v_{0}=3$ & $v_{0}=10$ & ML & $v_{0}=1$ & $v_{0}=3$ & $v_{0}=10$ & ML & $v_{0}=1$ & $v_{0}=3$ & $v_{0}=10$ \\
\hline \multirow{4}{*}{$\hat{\sigma}_{T}^{2}$} & & Ratio o & f Stable & Trait to & Autoregr & essive & Trait I & ariance & of $1: 1$ & & & & \\
\hline & .2 & 0.00 & -0.01 & -0.01 & -0.02 & 0.06 & 0.06 & 0.06 & 0.06 & 94.1 & 94.3 & 93.8 & 93.6 \\
\hline & .5 & 0.00 & -0.01 & -0.01 & -0.01 & 0.07 & 0.07 & 0.07 & 0.07 & 93.5 & 93.3 & 93.8 & 93.9 \\
\hline & .8 & -0.03 & 0.01 & 0.04 & 0.11 & 0.19 & 0.15 & 0.13 & 0.15 & 95.9 & 94.4 & 94.6 & 87.4 \\
\hline \multirow{3}{*}{$\hat{\sigma}_{A}^{2}$} & .2 & 0.14 & -0.23 & -0.33 & -0.38 & 0.52 & 0.38 & 0.37 & 0.39 & 86.1 & 94.6 & 89.7 & 48.9 \\
\hline & .5 & 0.02 & 0.01 & 0.01 & 0.00 & 0.10 & 0.09 & 0.09 & 0.08 & 95.9 & 94.9 & 95.5 & 96.5 \\
\hline & .8 & 0.03 & -0.02 & -0.04 & -0.09 & 0.16 & 0.12 & 0.10 & 0.11 & 92.8 & 94.8 & 94.1 & 84.5 \\
\hline \multirow{3}{*}{$\hat{\sigma}_{S}^{2}$} & .2 & -0.16 & 0.28 & 0.39 & 0.46 & 0.62 & 0.45 & 0.44 & 0.47 & 86.7 & 94.7 & 89.3 & 44.6 \\
\hline & .5 & -0.02 & -0.01 & 0.00 & 0.01 & 0.13 & 0.12 & 0.11 & 0.09 & 96.2 & 94.7 & 95.5 & 97.3 \\
\hline & .8 & 0.00 & 0.00 & -0.01 & -0.04 & 0.04 & 0.04 & 0.04 & 0.05 & 94.0 & 94.7 & 94.6 & 86.3 \\
\hline \multirow{3}{*}{$\hat{\beta}$} & .2 & 0.12 & 0.14 & 0.38 & 0.67 & 0.58 & 0.51 & 0.54 & 0.71 & 96.0 & 94.8 & 90.9 & 31.4 \\
\hline & .5 & 0.00 & -0.01 & 0.00 & 0.00 & 0.12 & 0.13 & 0.11 & 0.09 & 94.4 & 94.5 & 95.4 & 97.5 \\
\hline & .8 & 0.00 & 0.00 & -0.02 & -0.06 & 0.06 & 0.06 & 0.06 & 0.08 & 93.4 & 93.7 & 93.5 & 76.5 \\
\hline \multirow{4}{*}{$\hat{\sigma}_{T}^{2}$} & & Ratio o & f Stable & Trait to & Autoregr & essive & Trait I & ariance & of $3: 1$ & & & & \\
\hline & .2 & 0.00 & -0.01 & -0.01 & -0.01 & 0.06 & 0.05 & 0.05 & 0.05 & 94.7 & 94.6 & 94.7 & 93.7 \\
\hline & .5 & -0.01 & -0.01 & -0.01 & -0.01 & 0.05 & 0.05 & 0.05 & 0.05 & 94.9 & 94.7 & 94.5 & 94.9 \\
\hline & .8 & -0.04 & 0.00 & 0.02 & 0.04 & 0.15 & 0.08 & 0.07 & 0.07 & 98.0 & 96.2 & 96.2 & 92.6 \\
\hline \multirow{3}{*}{$\hat{\sigma}_{A}^{2}$} & .2 & 0.37 & -0.29 & -0.28 & -0.21 & 0.94 & 0.42 & 0.33 & 0.23 & 84.9 & 97.3 & 98.5 & 99.1 \\
\hline & .5 & 0.04 & 0.02 & 0.03 & 0.05 & 0.16 & 0.14 & 0.13 & 0.11 & 96.9 & 95.3 & 95.5 & 95.4 \\
\hline & .8 & 0.11 & -0.02 & -0.04 & -0.06 & 0.38 & 0.14 & 0.11 & 0.10 & 96.2 & 96.2 & 97.3 & 95.5 \\
\hline \multirow{3}{*}{$\hat{\sigma}_{S}^{2}$} & .2 & -0.21 & 0.18 & 0.18 & 0.14 & 0.57 & 0.26 & 0.20 & 0.15 & 85.2 & 96.1 & 98.2 & 98.6 \\
\hline & .5 & -0.02 & 0.00 & 0.00 & -0.02 & 0.11 & 0.10 & 0.09 & 0.07 & 93.4 & 94.4 & 96.0 & 96.9 \\
\hline & .8 & 0.00 & 0.00 & -0.01 & -0.04 & 0.04 & 0.04 & 0.04 & 0.05 & 95.7 & 96.0 & 94.9 & 80.5 \\
\hline \multirow{3}{*}{$\hat{\beta}$} & .2 & 0.19 & 0.09 & 0.23 & 0.43 & 0.84 & 0.61 & 0.46 & 0.47 & 94.3 & 97.0 & 97.1 & 83.8 \\
\hline & .5 & 0.00 & -0.01 & -0.02 & -0.04 & 0.18 & 0.18 & 0.16 & 0.12 & 95.1 & 95.1 & 95.7 & 98.4 \\
\hline & .8 & 0.00 & -0.01 & -0.03 & -0.10 & 0.08 & 0.08 & 0.08 & 0.12 & 94.5 & 95.8 & 94.6 & 73.4 \\
\hline
\end{tabular}

Note. $\mathrm{RMSE}=$ root mean square error. $\mathrm{ML}=$ Maximum Likelihood with constrained estimation. $v_{0}$ $=$ prior sample size for prior distributions. $\beta=$ stability parameter. Relative biases smaller than -.10 or larger than .10 and coverage rates smaller than $91 \%$ and larger than $98 \%$ are written in bold. 
Table A18

Relative Bias, Relative RMSE, and Coverage for the Maximum Likelihood and the Bayesian Approach (with Different Priors) as a Function of Measurement Waves $W$ and the Population State Variance $\sigma_{S}^{2}$ for a Medium Sample Size $(N=500)$, Ratio of Stable Trait Variance to Autoregressive Variance of $1: 1$ and a Stability Parameter.5

\begin{tabular}{|c|c|c|c|c|c|c|c|c|c|c|c|c|c|c|}
\hline & \multirow[b]{2}{*}{$W$} & \multirow[b]{2}{*}{$\sigma_{S}^{2}$} & \multicolumn{4}{|c|}{ Relative bias } & \multicolumn{4}{|c|}{ Relative RMSE } & \multicolumn{4}{|c|}{ Coverage } \\
\hline & & & ML & $v_{0}=1$ & $v_{0}=3$ & $v_{0}=10$ & ML & $v_{0}=1$ & $v_{0}=3$ & $v_{0}=10$ & ML & $v_{0}=1$ & $v_{0}=3$ & $v_{0}=10$ \\
\hline \multirow{6}{*}{$\hat{\sigma}_{T}^{2}$} & 4 & .1 & -0.12 & -0.06 & -0.07 & -0.06 & 0.35 & 0.17 & 0.16 & 0.14 & 97.6 & 94.1 & 93.4 & 95.3 \\
\hline & 4 & .3 & -0.24 & 0.02 & 0.01 & -0.01 & 0.52 & 0.16 & 0.14 & 0.12 & 96.1 & 98.9 & 98.9 & 99.0 \\
\hline & 6 & .1 & -0.02 & -0.04 & -0.05 & -0.07 & 0.12 & 0.11 & 0.12 & 0.12 & 95.8 & 93.3 & 92.1 & 90.9 \\
\hline & 6 & .3 & -0.04 & -0.01 & -0.02 & -0.02 & 0.18 & 0.12 & 0.11 & 0.10 & 97.5 & 96.9 & 97.3 & 97.0 \\
\hline & 9 & .1 & 0.00 & -0.01 & -0.02 & -0.04 & 0.09 & 0.09 & 0.09 & 0.09 & 95.1 & 95.1 & 94.6 & 94.3 \\
\hline & 9 & .3 & 0.00 & 0.00 & 0.00 & -0.01 & 0.10 & 0.10 & 0.10 & 0.09 & 94.9 & 94.7 & 94.9 & 94.8 \\
\hline \multirow{6}{*}{$\hat{\sigma}_{A}^{2}$} & 4 & .1 & 0.14 & 0.01 & -0.03 & -0.11 & 0.28 & 0.14 & 0.14 & 0.16 & 98.7 & 96.0 & 97.8 & 95.9 \\
\hline & 4 & .3 & 0.41 & 0.09 & 0.04 & -0.01 & 0.54 & 0.26 & 0.22 & 0.17 & 99.3 & 95.4 & 96.5 & 97.4 \\
\hline & 6 & .1 & 0.03 & 0.00 & -0.03 & -0.07 & 0.10 & 0.08 & 0.08 & 0.11 & 97.0 & 97.3 & 95.2 & 88.7 \\
\hline & 6 & .3 & 0.11 & 0.06 & 0.04 & 0.01 & 0.25 & 0.17 & 0.14 & 0.12 & 98.7 & 94.6 & 96.6 & 98.0 \\
\hline & 9 & .1 & 0.01 & -0.01 & -0.03 & -0.07 & 0.08 & 0.06 & 0.06 & 0.09 & 96.2 & 97.2 & 94.5 & 78.6 \\
\hline & 9 & .3 & 0.04 & 0.03 & 0.01 & 0.00 & 0.15 & 0.13 & 0.11 & 0.09 & 95.8 & 95.7 & 96.9 & 97.8 \\
\hline \multirow{5}{*}{$\hat{\sigma}_{S}^{2}$} & 4 & .1 & -0.08 & 0.45 & 0.55 & 0.69 & 0.76 & 0.59 & 0.60 & 0.71 & 91.6 & 98.9 & 88.8 & 4.5 \\
\hline & 4 & .3 & -0.20 & 0.10 & 0.08 & 0.05 & 0.54 & 0.23 & 0.17 & 0.12 & 91.0 & 99.7 & 99.7 & 99.8 \\
\hline & 6 & .1 & -0.10 & 0.19 & 0.36 & 0.55 & 0.55 & 0.39 & 0.44 & 0.58 & 94.6 & 97.5 & 90.7 & 16.4 \\
\hline & 6 & .3 & -0.07 & 0.03 & 0.04 & 0.03 & 0.32 & 0.21 & 0.16 & 0.11 & 94.3 & 98.0 & 99.2 & 100 \\
\hline & 9 & .1 & -0.03 & 0.10 & 0.24 & 0.44 & 0.39 & 0.30 & 0.32 & 0.46 & 94.6 & 97.1 & 91.4 & 34.6 \\
\hline \multirow{7}{*}{$\hat{\beta}$} & 9 & .3 & -0.04 & -0.01 & 0.00 & 0.01 & 0.19 & 0.17 & 0.14 & 0.11 & 95.4 & 95.8 & 97.8 & 99.0 \\
\hline & 4 & .1 & 0.05 & 0.18 & 0.20 & 0.20 & 0.33 & 0.29 & 0.24 & 0.21 & 96.1 & 96.9 & 93.3 & 86.8 \\
\hline & 4 & .3 & 0.04 & 0.01 & 0.01 & 0.02 & 0.51 & 0.35 & 0.17 & 0.06 & 92.9 & 100 & 100 & 100 \\
\hline & 6 & .1 & -0.01 & 0.05 & 0.11 & 0.16 & 0.18 & 0.14 & 0.15 & 0.18 & 97.3 & 97.5 & 92.6 & 71.8 \\
\hline & 6 & .3 & 0.01 & 0.00 & 0.01 & 0.01 & 0.28 & 0.27 & 0.18 & 0.09 & 94.9 & 98.5 & 99.7 & 100 \\
\hline & 9 & .1 & 0.00 & 0.02 & 0.06 & 0.11 & 0.11 & 0.09 & 0.10 & 0.13 & 94.0 & 96.6 & 91.9 & 69.0 \\
\hline & 9 & .3 & -0.01 & -0.02 & -0.01 & 0.00 & 0.16 & 0.16 & 0.14 & 0.09 & 95.1 & 95.4 & 97.7 & 99.3 \\
\hline
\end{tabular}

Note. $\mathrm{RMSE}=$ root mean square error. $\mathrm{ML}=$ Maximum Likelihood with constrained estimation. $v_{0}=$ prior sample size for prior distributions. $W=$ measurement waves. $\sigma_{S}^{2}=$ true state variance. Relative biases smaller than -.10 or larger than .10 and coverage rates smaller than $91 \%$ and larger than $98 \%$ are written in bold. 
Table A19

Relative Bias, Relative RMSE, and Coverage for the Maximum Likelihood and the Bayesian Approach (with Different Priors) as a Function of Measurement Waves $W$ and the Population State Variance $\sigma_{S}^{2}$ for a Small Sample Size $(N=200)$, Ratio of Stable Trait Variance to Autoregressive Variance of 1:1 and a Stability Parameter.5

\begin{tabular}{|c|c|c|c|c|c|c|c|c|c|c|c|c|c|c|}
\hline & \multirow[b]{2}{*}{$W$} & \multirow[b]{2}{*}{$\sigma_{S}^{2}$} & \multicolumn{4}{|c|}{ Relative bias } & \multicolumn{4}{|c|}{ Relative RMSE } & \multicolumn{4}{|c|}{ Coverage } \\
\hline & & & ML & $v_{0}=1$ & $v_{0}=3$ & $v_{0}=10$ & ML & $v_{0}=1$ & $v_{0}=3$ & $v_{0}=10$ & ML & $v_{0}=1$ & $v_{0}=3$ & $v_{0}=10$ \\
\hline \multirow{6}{*}{$\hat{\boldsymbol{\sigma}}_{T}^{2}$} & 4 & .1 & -0.22 & -0.10 & -0.08 & -0.05 & 0.50 & 0.24 & 0.21 & 0.16 & 99.0 & 96.1 & 96.5 & 97.4 \\
\hline & 4 & .3 & -0.29 & 0.00 & -0.02 & -0.03 & 0.60 & 0.21 & 0.18 & 0.15 & 97.3 & 99.1 & 99.1 & 99.1 \\
\hline & 6 & .1 & -0.04 & -0.07 & -0.08 & -0.08 & 0.21 & 0.18 & 0.17 & 0.16 & 98.0 & 95.4 & 95.3 & 95.6 \\
\hline & 6 & .3 & -0.12 & -0.02 & -0.02 & -0.02 & 0.38 & 0.19 & 0.18 & 0.15 & 97.5 & 96.2 & 96.4 & 97.1 \\
\hline & 9 & .1 & -0.01 & -0.04 & -0.05 & -0.07 & 0.14 & 0.14 & 0.14 & 0.14 & 95.3 & 95.1 & 94.5 & 93.9 \\
\hline & 9 & .3 & -0.02 & -0.03 & -0.03 & -0.03 & 0.18 & 0.16 & 0.15 & 0.14 & 94.3 & 94.1 & 94.6 & 95.4 \\
\hline \multirow{6}{*}{$\hat{\sigma}_{A}^{2}$} & 4 & .1 & 0.24 & -0.01 & -0.08 & -0.20 & 0.41 & 0.20 & 0.19 & 0.23 & 99.2 & 98.0 & 98.5 & 91.0 \\
\hline & 4 & .3 & 0.53 & 0.08 & 0.02 & -0.04 & 0.65 & 0.34 & 0.27 & 0.18 & 99.8 & 97.0 & 98.4 & 99.7 \\
\hline & 6 & .1 & 0.06 & -0.01 & -0.05 & -0.12 & 0.17 & 0.12 & 0.13 & 0.16 & 98.1 & 97.3 & 97.1 & 89.6 \\
\hline & 6 & .3 & 0.27 & 0.07 & 0.02 & -0.02 & 0.45 & 0.24 & 0.20 & 0.15 & 99.6 & 96.9 & 98.0 & 98.2 \\
\hline & 9 & .1 & 0.02 & -0.02 & -0.05 & -0.11 & 0.11 & 0.09 & 0.10 & 0.13 & 95.8 & 97.5 & 93.1 & 81.8 \\
\hline & 9 & .3 & 0.09 & 0.04 & 0.02 & -0.01 & 0.25 & 0.18 & 0.15 & 0.12 & 97.8 & 97.2 & 98.0 & 99.0 \\
\hline \multirow{5}{*}{$\hat{\sigma}_{S}^{2}$} & 4 & .1 & -0.04 & 0.54 & 0.65 & 0.84 & 0.86 & 0.68 & 0.72 & 0.87 & 91.4 & 99.8 & 87.9 & 1.3 \\
\hline & 4 & .3 & -0.28 & 0.10 & 0.07 & 0.05 & 0.64 & 0.27 & 0.20 & 0.14 & 91.7 & 99.9 & 100 & 100 \\
\hline & 6 & .1 & -0.07 & 0.36 & 0.51 & 0.71 & 0.68 & 0.53 & 0.58 & 0.73 & 93.8 & 97.5 & 89.2 & 5.2 \\
\hline & 6 & .3 & -0.18 & 0.05 & 0.05 & 0.04 & 0.51 & 0.26 & 0.19 & 0.13 & 91.8 & 99.0 & 99.3 & 99.7 \\
\hline & 9 & .1 & -0.07 & 0.20 & 0.39 & 0.61 & 0.54 & 0.39 & 0.47 & 0.63 & 94.7 & 97.8 & 90.8 & 10.1 \\
\hline \multirow{7}{*}{$\hat{\beta}$} & 9 & .3 & -0.08 & 0.01 & 0.02 & 0.03 & 0.32 & 0.23 & 0.17 & 0.11 & 94.7 & 97.5 & 98.9 & 99.8 \\
\hline & 4 & .1 & 0.10 & 0.25 & 0.23 & 0.18 & 0.41 & 0.33 & 0.26 & 0.19 & 95.0 & 97.6 & 97.1 & 98.8 \\
\hline & 4 & .3 & 0.03 & -0.01 & 0.01 & 0.01 & 0.57 & 0.31 & 0.13 & 0.06 & 95.9 & 100 & 100 & 100 \\
\hline & 6 & .1 & 0.03 & 0.12 & 0.17 & 0.18 & 0.24 & 0.21 & 0.20 & 0.20 & 96.9 & 96.0 & 92.1 & 83.7 \\
\hline & 6 & .3 & 0.00 & -0.01 & 0.00 & 0.01 & 0.43 & 0.32 & 0.19 & 0.08 & 91.0 & 99.4 & 100 & 100 \\
\hline & 9 & .1 & 0.00 & 0.05 & 0.10 & 0.15 & 0.16 & 0.13 & 0.14 & 0.16 & 96.6 & 96.8 & 93.0 & 75.5 \\
\hline & 9 & .3 & 0.00 & -0.02 & 0.00 & 0.01 & 0.26 & 0.24 & 0.17 & 0.10 & 94.8 & 97.8 & 99.3 & 99.9 \\
\hline
\end{tabular}

Note. $\mathrm{RMSE}=$ root mean square error. $\mathrm{ML}=$ Maximum Likelihood with constrained estimation. $v_{0}=$ prior sample size for prior distributions. $W=$ measurement waves. $\sigma_{S}^{2}=$ true state variance. Relative biases smaller than -.10 or larger than .10 and coverage rates smaller than $91 \%$ and larger than $98 \%$ are written in bold. 
Table A20

Relative Bias, Relative RMSE, and Coverage for the Maximum Likelihood and the Bayesian Approach (with Different Priors) as a Function of Measurement Waves $W$ and the Population State Variance $\sigma_{S}^{2}$ for a Large Sample Size $(N=1000)$, Ratio of Stable Trait Variance to Autoregressive Variance of 1:1 and a Stability Parameter .5

\begin{tabular}{|c|c|c|c|c|c|c|c|c|c|c|c|c|c|c|}
\hline & \multirow[b]{2}{*}{$W$} & \multirow[b]{2}{*}{$\sigma_{S}^{2}$} & \multicolumn{4}{|c|}{ Relative bias } & \multicolumn{4}{|c|}{ Relative RMSE } & \multicolumn{4}{|c|}{ Coverage } \\
\hline & & & ML & $v_{0}=1$ & $v_{0}=3$ & $v_{0}=10$ & ML & $v_{0}=1$ & $v_{0}=3$ & $v_{0}=10$ & ML & $v_{0}=1$ & $v_{0}=3$ & $v_{0}=10$ \\
\hline \multirow{6}{*}{$\hat{\sigma}_{T}^{2}$} & 4 & .1 & -0.07 & -0.05 & -0.07 & -0.08 & 0.25 & 0.15 & 0.14 & 0.12 & 97.8 & 93.7 & 92.1 & 90.9 \\
\hline & 4 & .3 & -0.16 & 0.03 & 0.02 & 0.00 & 0.42 & 0.13 & 0.11 & 0.09 & 94.2 & 98.5 & 98.9 & 98.7 \\
\hline & 6 & .1 & -0.01 & -0.02 & -0.04 & -0.05 & 0.08 & 0.08 & 0.08 & 0.09 & 95.6 & 94.8 & 93.7 & 92.3 \\
\hline & 6 & .3 & -0.02 & 0.00 & -0.01 & -0.01 & 0.13 & 0.10 & 0.09 & 0.08 & 97.0 & 95.3 & 96.7 & 97.7 \\
\hline & 9 & .1 & 0.00 & -0.01 & -0.02 & -0.03 & 0.06 & 0.06 & 0.06 & 0.06 & 95.8 & 96.0 & 95.5 & 94.5 \\
\hline & 9 & .3 & 0.00 & -0.01 & -0.01 & -0.01 & 0.07 & 0.07 & 0.07 & 0.07 & 93.5 & 93.3 & 93.8 & 93.9 \\
\hline \multirow{6}{*}{$\hat{\sigma}_{A}^{2}$} & 4 & .1 & 0.09 & 0.02 & 0.00 & -0.05 & 0.20 & 0.11 & 0.10 & 0.10 & 98.8 & 96.7 & 97.3 & 96.6 \\
\hline & 4 & .3 & 0.29 & 0.08 & 0.05 & 0.01 & 0.43 & 0.19 & 0.16 & 0.13 & 99.1 & 94.4 & 96.1 & 96.9 \\
\hline & 6 & .1 & 0.02 & 0.00 & -0.02 & -0.05 & 0.07 & 0.06 & 0.06 & 0.07 & 96.7 & 97.5 & 95.7 & 88.6 \\
\hline & 6 & .3 & 0.06 & 0.04 & 0.03 & 0.01 & 0.18 & 0.13 & 0.11 & 0.09 & 97.3 & 95.6 & 96.7 & 97.5 \\
\hline & 9 & .1 & 0.01 & 0.00 & -0.02 & -0.05 & 0.06 & 0.05 & 0.05 & 0.06 & 95.1 & 96.2 & 94.6 & 80.5 \\
\hline & 9 & .3 & 0.02 & 0.01 & 0.01 & 0.00 & 0.10 & 0.09 & 0.09 & 0.08 & 95.9 & 94.9 & 95.5 & 96.5 \\
\hline \multirow{5}{*}{$\hat{\sigma}_{S}^{2}$} & 4 & .1 & -0.08 & 0.35 & 0.46 & 0.59 & 0.65 & 0.51 & 0.52 & 0.61 & 92.5 & 96.7 & 89.6 & 9.7 \\
\hline & 4 & .3 & -0.15 & 0.08 & 0.06 & 0.04 & 0.46 & 0.21 & 0.15 & 0.10 & 92.1 & 99.7 & 99.9 & 99.9 \\
\hline & 6 & .1 & -0.05 & 0.12 & 0.26 & 0.44 & 0.42 & 0.31 & 0.34 & 0.46 & 95.3 & 96.4 & 92.4 & 34.1 \\
\hline & 6 & .3 & -0.05 & 0.01 & 0.02 & 0.03 & 0.24 & 0.19 & 0.15 & 0.10 & 93.7 & 94.5 & 97.2 & 99.1 \\
\hline & 9 & .1 & -0.04 & 0.04 & 0.14 & 0.31 & 0.28 & 0.23 & 0.22 & 0.33 & 95.5 & 97.2 & 94.5 & 58.1 \\
\hline \multirow{7}{*}{$\hat{\beta}$} & 9 & .3 & -0.02 & -0.01 & 0.00 & 0.01 & 0.13 & 0.12 & 0.11 & 0.09 & 96.2 & 94.7 & 95.5 & 97.3 \\
\hline & 4 & .1 & 0.03 & 0.13 & 0.17 & 0.21 & 0.27 & 0.24 & 0.22 & 0.22 & 97.6 & 95.7 & 90.8 & 72.5 \\
\hline & 4 & .3 & 0.03 & 0.01 & 0.00 & 0.02 & 0.44 & 0.35 & 0.19 & 0.08 & 92.7 & 100 & 100 & 100 \\
\hline & 6 & .1 & 0.00 & 0.03 & 0.08 & 0.14 & 0.13 & 0.11 & 0.12 & 0.15 & 94.9 & 96.0 & 91.9 & 64.4 \\
\hline & 6 & .3 & 0.00 & -0.01 & 0.00 & 0.01 & 0.23 & 0.23 & 0.17 & 0.11 & 93.2 & 94.4 & 97.5 & 99.7 \\
\hline & 9 & .1 & -0.01 & 0.01 & 0.03 & 0.08 & 0.08 & 0.07 & 0.07 & 0.09 & 96.0 & 97.4 & 94.8 & 72.9 \\
\hline & 9 & .3 & 0.00 & -0.01 & 0.00 & 0.00 & 0.12 & 0.13 & 0.11 & 0.09 & 94.4 & 94.5 & 95.4 & 97.5 \\
\hline
\end{tabular}

Note. $\mathrm{RMSE}=$ root mean square error. $\mathrm{ML}=$ Maximum Likelihood with constrained estimation. $v_{0}=$ prior sample size for prior distributions. $W=$ measurement waves. $\sigma_{S}^{2}=$ true state variance. Relative biases smaller than -.10 or larger than .10 and coverage rates smaller than $91 \%$ and larger than $98 \%$ are written in bold. 

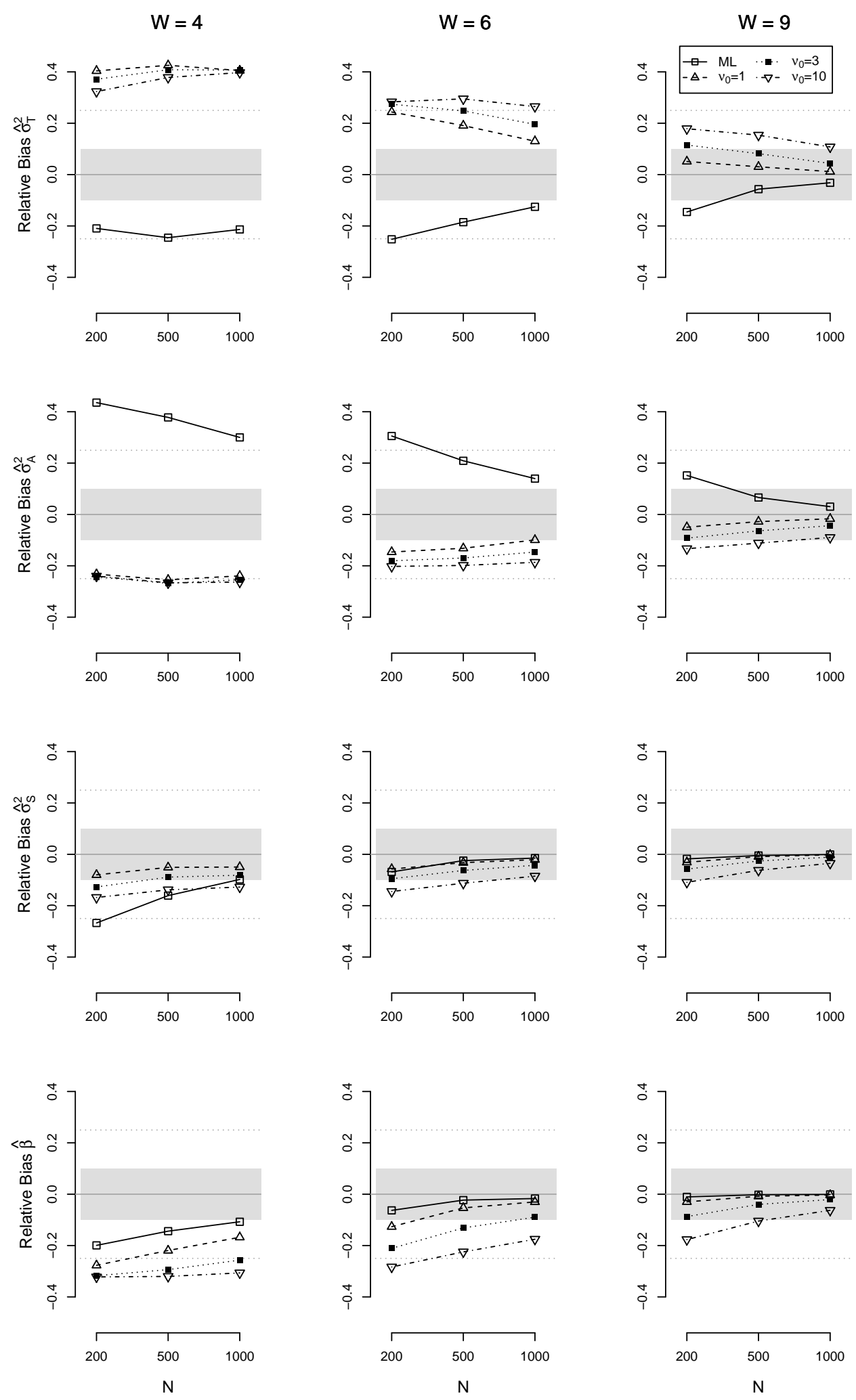

Figure A1. Relative bias of the estimators of the STARTS model parameters for selected conditions in the simulation study. The ratio of stable trait variance to autoregressive trait variance was 1:1. The state variance was .3. The stability parameter was .8. $W=$ number of measurement waves. $N$ $=$ sample size. 

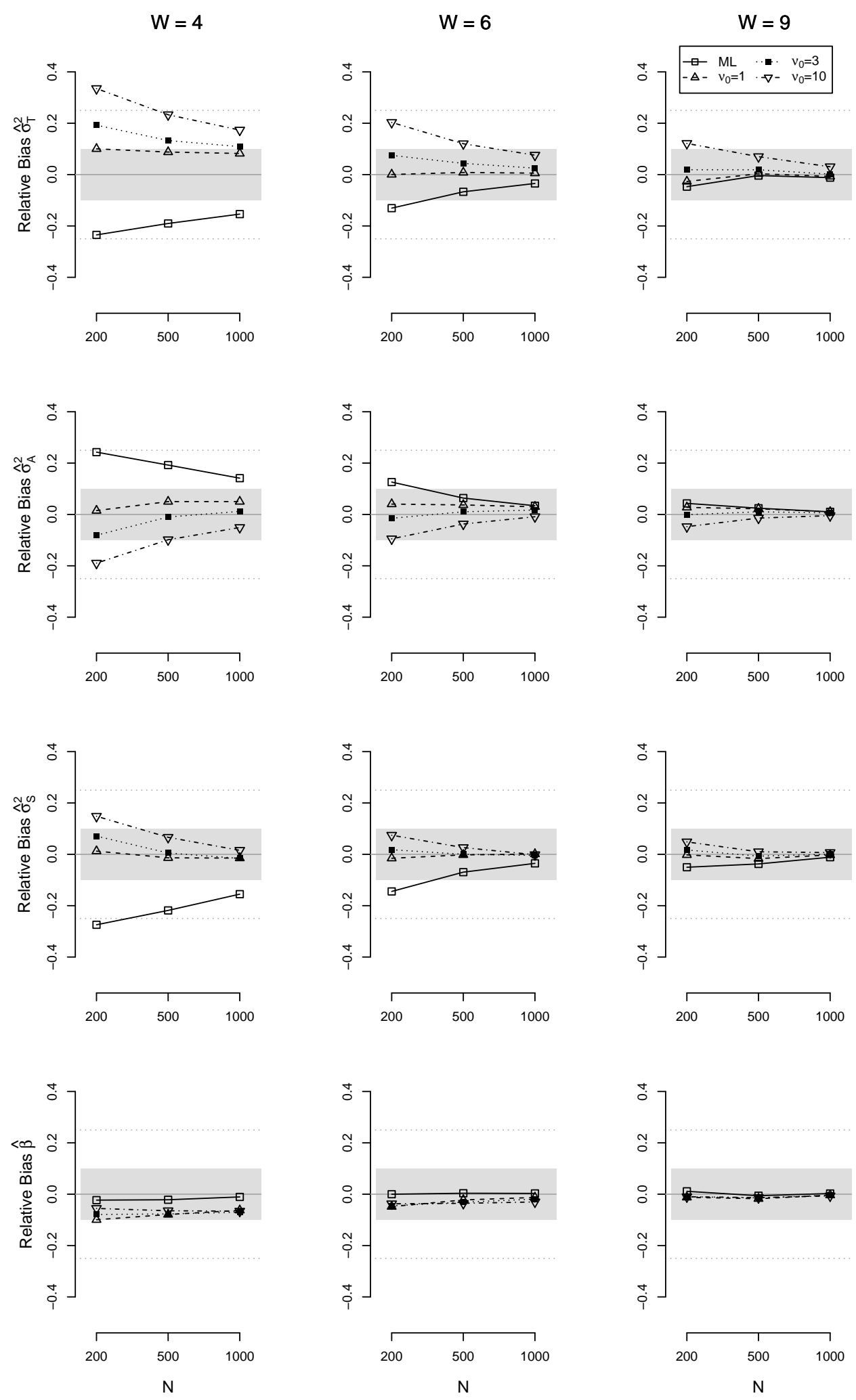

Figure A2. Relative bias of the estimators of the STARTS model parameters for selected conditions in the simulation study. The ratio of stable trait variance to autoregressive trait variance was 1:3. The state variance was .3. The stability parameter was .5. $W=$ number of measurement waves. $N$ $=$ sample size. 

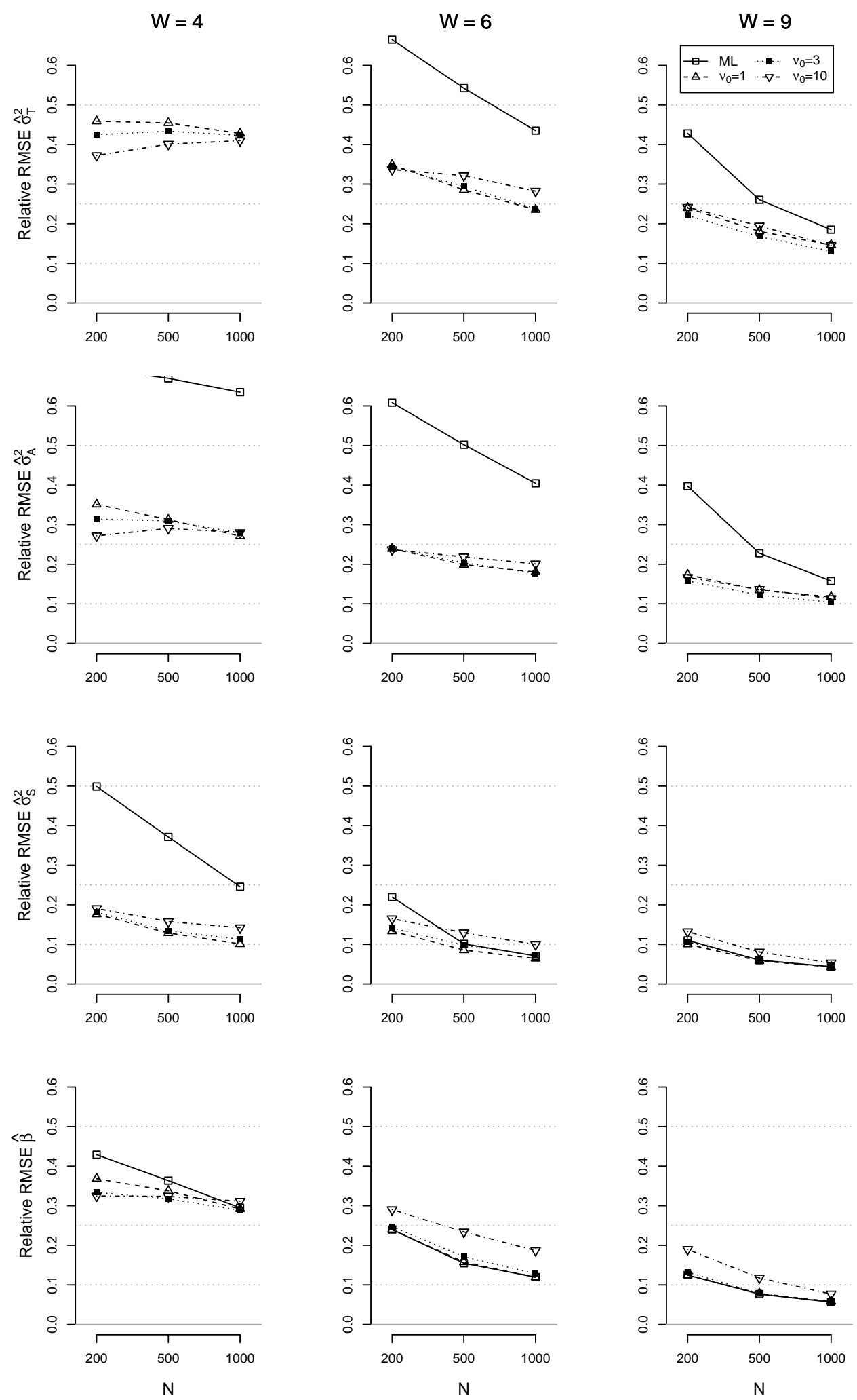

Figure A3. Relative RMSE (root mean square error) of the estimators of the STARTS model parameters for selected conditions in the simulation study. The ratio of stable trait variance to autoregressive trait variance was 1:1. The state variance was .3. The stability parameter was .8 . $W=$ number of measurement waves. $N=$ sample size. 

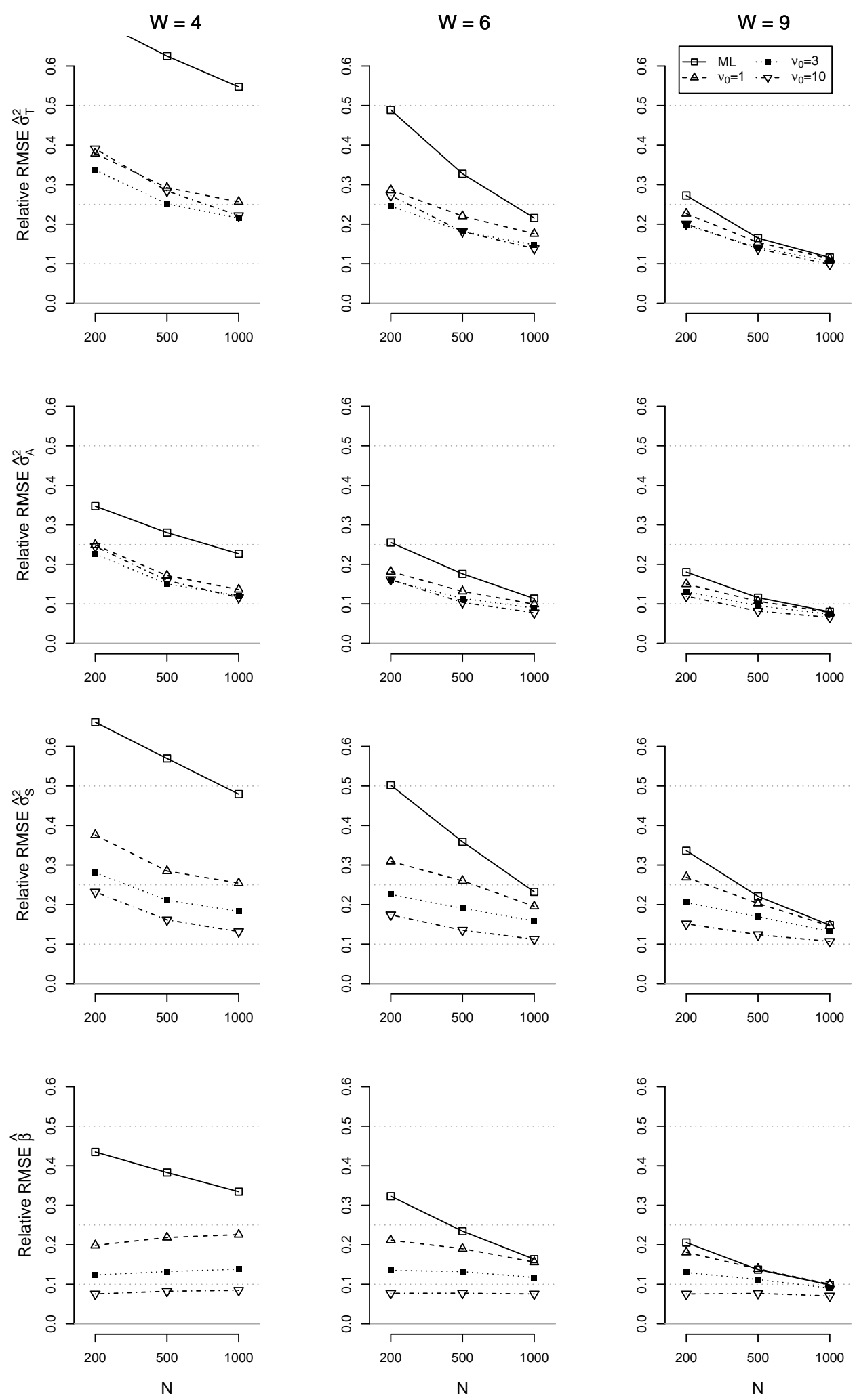

Figure A4. Relative RMSE (root mean square error) of the estimators of the STARTS model parameters for selected conditions in the simulation study. The ratio of stable trait variance to autoregressive trait variance was 1:3. The state variance was .3. The stability parameter was .5 . $W=$ number of measurement waves. $N=$ sample size. 

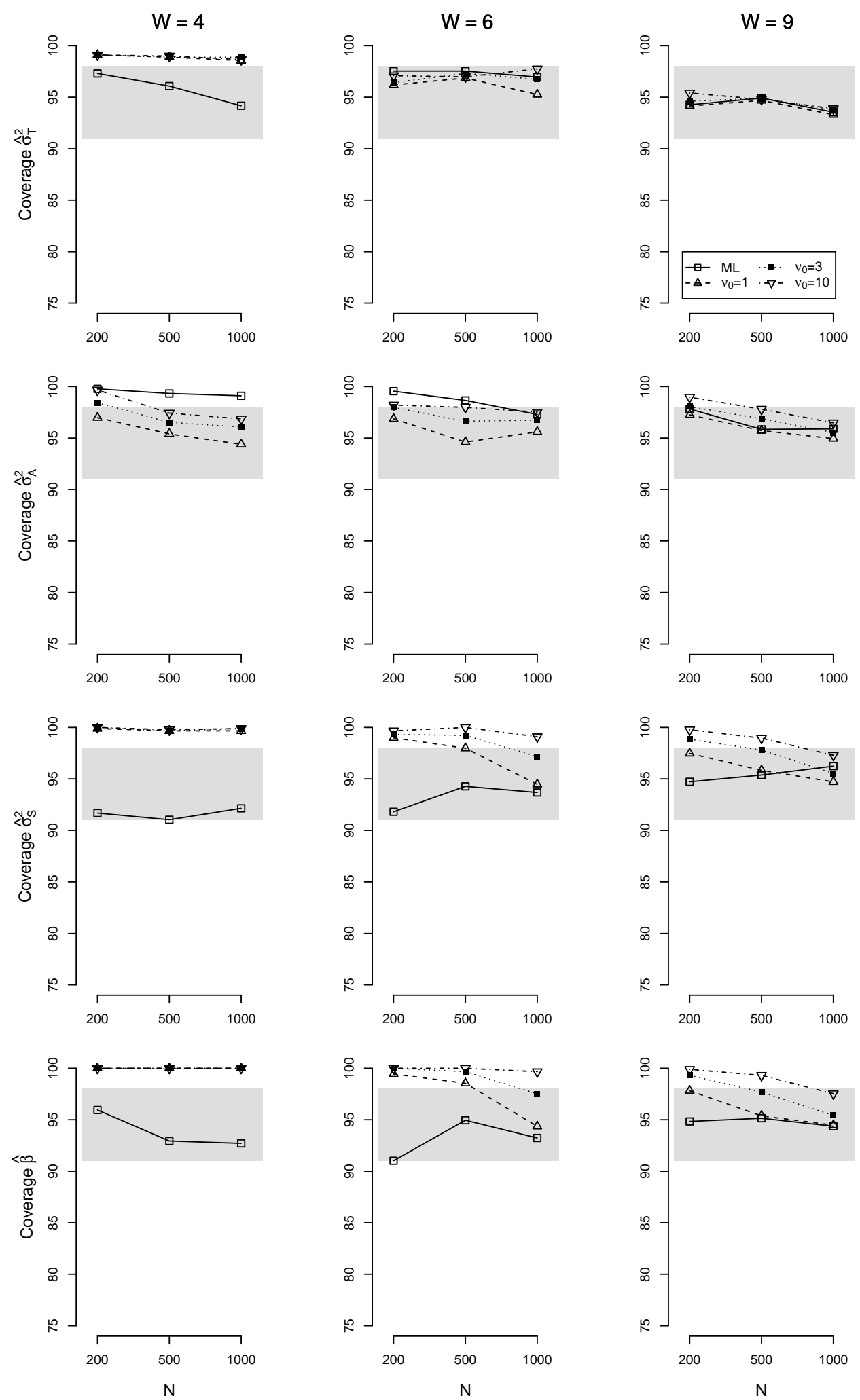

Figure A5. Coverage rates of the estimators of the trait variance and the state variance for selected conditions in the simulation study. The ratio of stable trait variance to autoregressive trait variance was 1:1. The state variance was .3. The stability parameter was .5. $W=$ number of measurement waves. $N=$ sample size. 

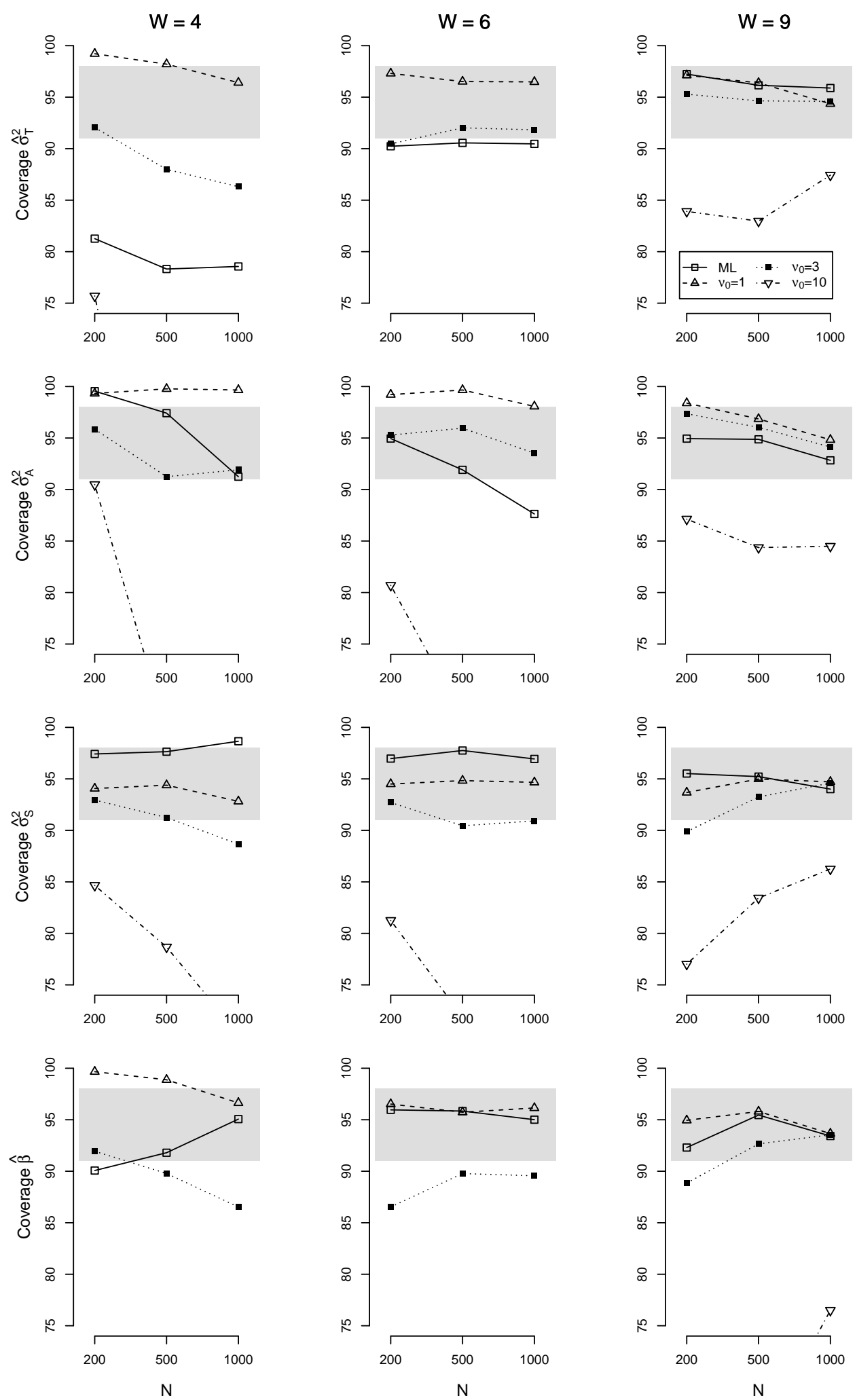

Figure A6. Coverage rates for selected conditions in the simulation study. The ratio of stable trait variance to autoregressive trait variance was 1:1. The state variance was .3. The stability parameter was .8. $W=$ number of measurement waves. $N=$ sample size. Coverage rates smaller than $75 \%$ are not displayed. In many conditions, coverage for the prior distribution with $v_{0}=10$ was below $75 \%$. 

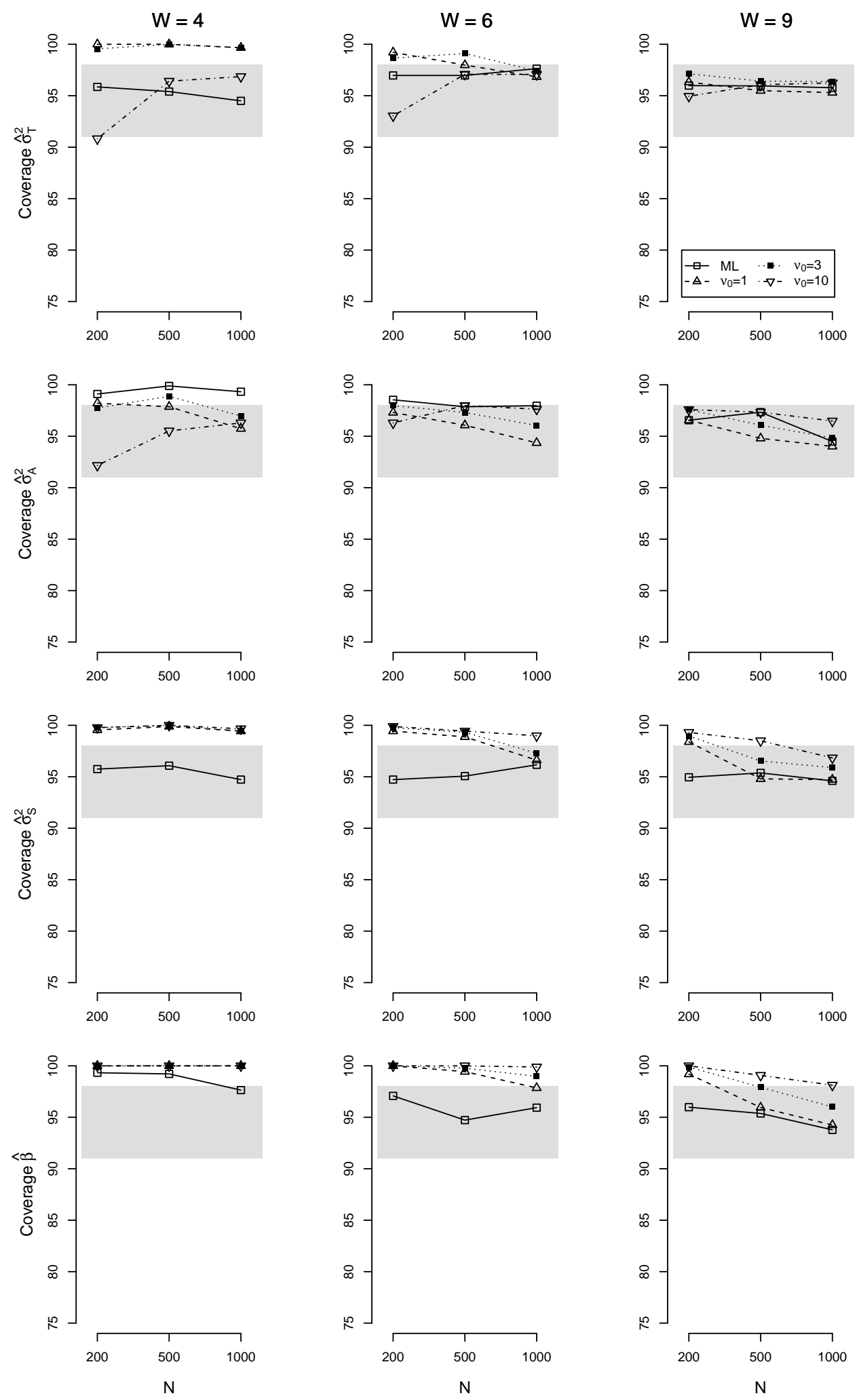

Figure A\%. Coverage rates for selected conditions in the simulation study. The ratio of stable trait variance to autoregressive trait variance was 1:3. The state variance was .3. The stability parameter was .5. $W=$ number of measurement waves. $N=$ sample size. 


\section{Supplement B: Additional Simulation for Large Sample Sizes}

In the main simulation study, the estimated relative RMSE of the estimator of the stability parameter $\hat{\beta}$, showed the unexpected tendency to increase when the sample size grew larger in conditions with $W=4$. We conducted a small additional simulation in which we also looked at very large sample sizes $(N=200,500,1,000,2,000,5,000$, and 10,000) for four-wave and six-wave designs with selected conditions from the main simulation. We held constant the trait variance (.35), the autoregressive trait variance (.35), the state variance (.30), and the stability parameter $(.5)$ of the data-generating model.

Figure B1 and B2 show the relative bias and relative RMSE for the estimators provided by ML with constrained estimation and the Bayesian approach with different prior specifications. As can be seen, with large sample sizes the estimated relative bias vanishes and all estimators were approximately unbiased (see Figure B1). In addition, the results for the estimated relative RMSE clearly show that there is a turning point and that the estimated relative RMSE of the estimator of the stability parameter decreased when the sample size increased from $N=1,000$ to $N=10,000$ (see Figure B2). Furthermore, with larger samples $(N \geq 2,000)$, the estimates produced by the ML approach closely matched the Bayesian estimates. 
$W=4$
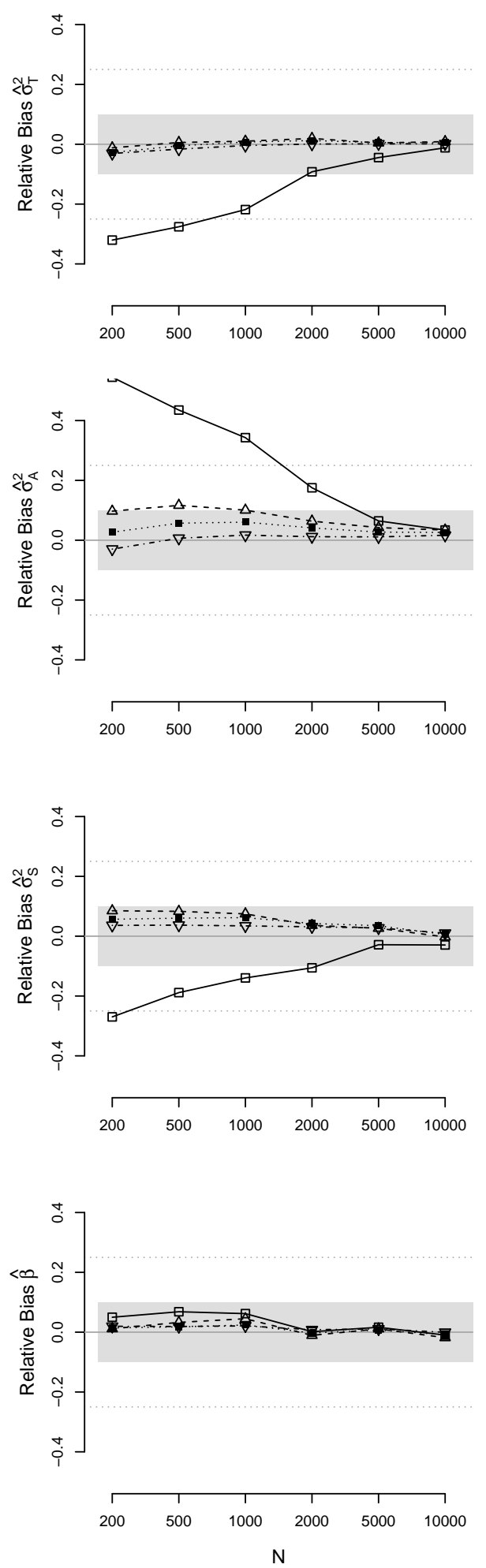

$W=6$
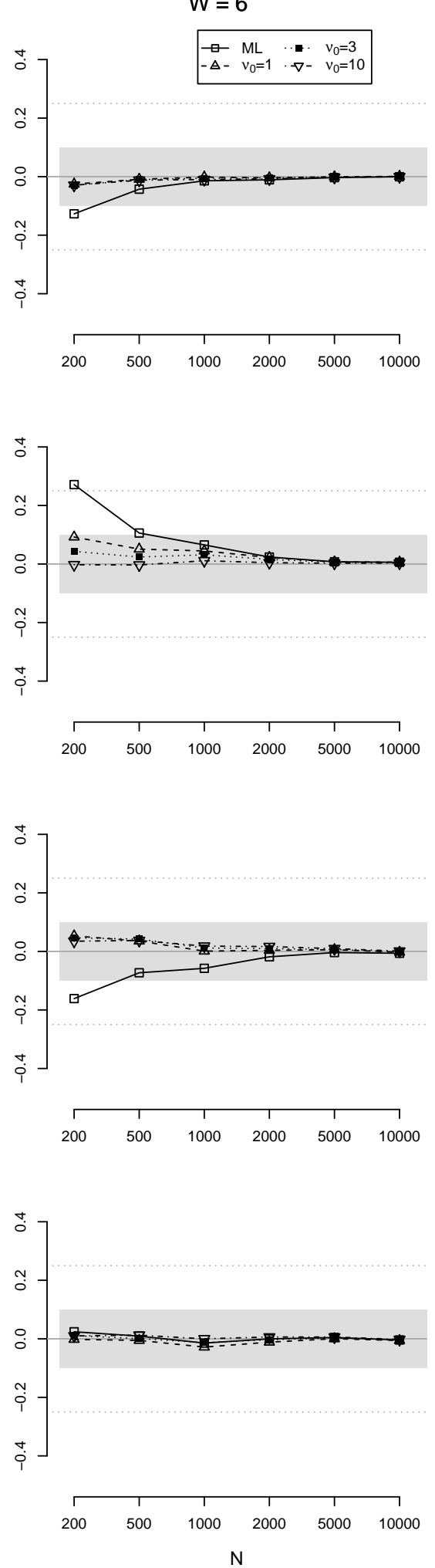

Figure B1. Relative bias of the estimators of the STARTS model parameters for selected conditions in the simulation study. The ratio of stable trait variance to autoregressive trait variance was 1:1. The state variance was .3. The stability parameter was .8. $\mathrm{W}=$ number of measurement waves. $N$ $=$ sample size. 

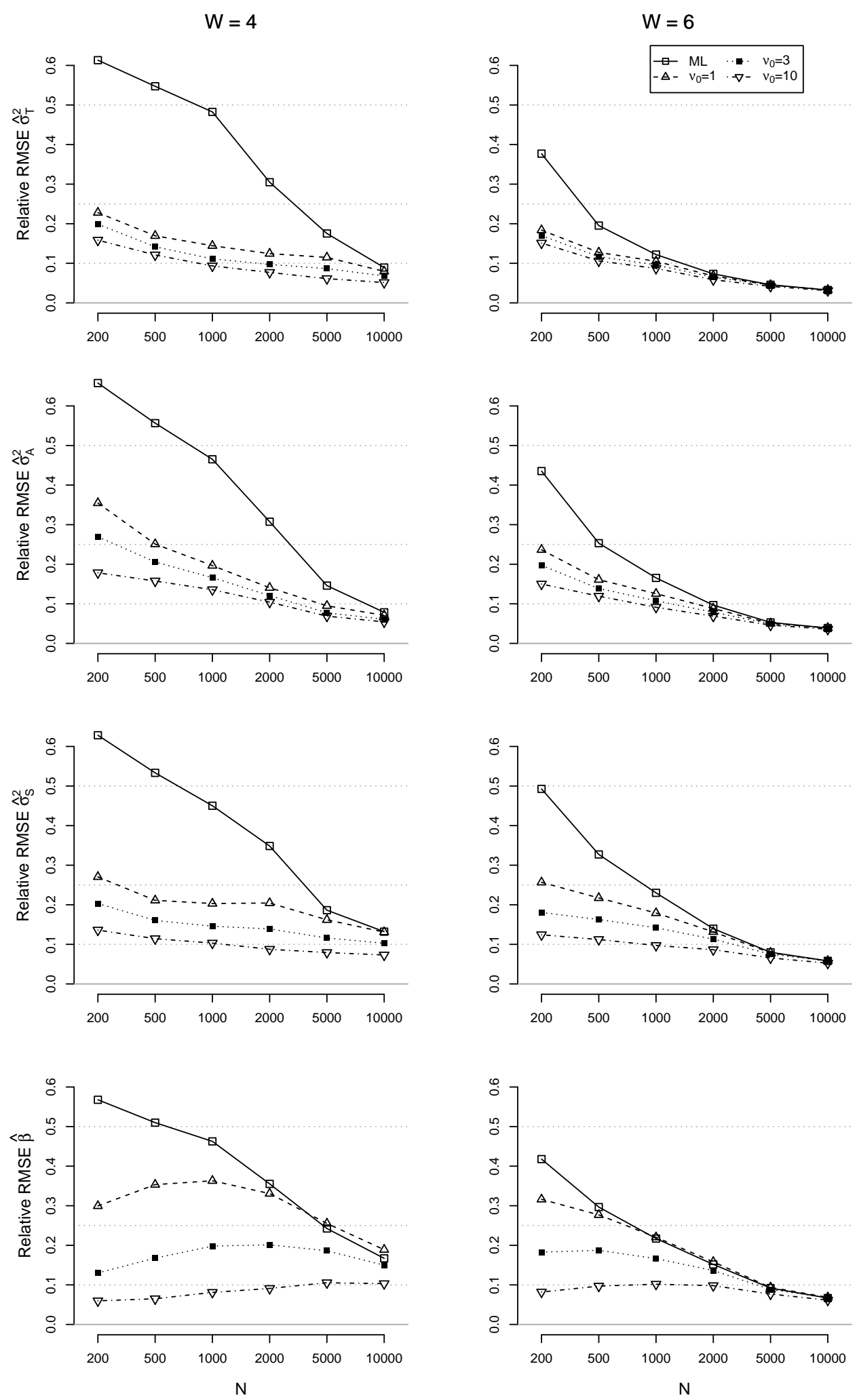

Figure B2. Relative RMSE of the estimators of the STARTS model parameters for selected conditions in the simulation study. The ratio of stable trait variance to autoregressive trait variance was 1:1. The state variance was .3. The stability parameter was .8. $W=$ number of measurement waves. $N$ $=$ sample size. 


\section{Supplement C: Additional Simulation on Impact of Misspecified Prior Distributions}

One crucial aspect of the Bayesian approach is the selection of prior distributions. To further investigate, how the results from the main simulation would change if prior information is inappropriately specified, we conducted an additional simulation study with selected conditions from the main simulation. We held constant the trait variance (.35), the autoregressive trait variance (.35), the state variance (.30), and the stability parameter (.5) of the data-generating model; and we varied the sample size $(N=200$ and 500) and number of measurement waves $(W=4,6$, and 9$)$.

We used three different types of prior distribution in the Bayesian approach. First, we included misspecified IG prior distributions that assumed a prior guess of $\sigma_{0, T}^{2}=.60$ for the stable trait variance, $\sigma_{0, A}^{2}=.20$ for the autoregressive trait variance, $\sigma_{0, S}^{2}=.20$ for the state factor variance, and $\beta_{0}=.5$ for the stability parameter with prior sample sizes of $v_{0}=1$ and $v_{0}=3$. Second, for comparison purposes, we specified uniform distributions that assumed that the STARTS model variances were uniformly distributed across the range from 0 to 10 and the stability parameter across the range from 0 to 1 . Third, we compared the performances of these prior distributions with the prior distributions that were used in the main simulation which assumed a prior guess of .33 for the stable trait variance, the autoregressive trait variance, and the state factor variance with prior sample sizes of $v_{0}=1$ and $v_{0}=3$. As the specification of the prior distributions in the main simulation nearly matched the true data generating parameters, these priors were labelled correctly specified prior distribution in the following results. We also included the Maximum Likelihood approach with constrained estimation.

Table C1 shows the relative bias and relative RMSE for the estimators provided by ML with constrained estimation and the Bayesian approach with correctly and misspecified prior distributions. The results showed that the models with misspecified priors were slightly more biased than the models with the correctly specified prior distributions but were still less biased than the estimates obtained from the ML approach. With regard to the relative RMSE, the models with misspecified prior distributions provided more accurate estimates than the ML approach. It is interesting that even the Bayesian approach with uniform prior distributions, which added no information to the likelihood, outperformed the ML approach.

It is also an interesting finding that in many conditions the Bayesian approach with misspecified prior distributions produced smaller relative RMSE values than the Bayesian approach with uniform prior distributions. Figure C1 further explores for the estimator of the state variance why the uniform prior distribution showed a poorer performance than the misspecified prior distributions in some of the conditions (condition with $N=200$ and $W=4$ ). As can be seen, the ML approach produced boundary estimates or very small variance estimates in about one third of the solutions. However, the occurrence of very small variance estimates is also not avoided in the Bayesian approach with a uniform distribution for the state variance. 
Table C1

Relative Bias and Relative RMSE for the Maximum Likelihood and the Bayesian Approach with Uninformative, Weakly Informative and Misspecified Priors

\begin{tabular}{|c|c|c|c|c|c|c|c|c|c|c|c|c|c|c|}
\hline & \multirow[b]{3}{*}{$W$} & \multirow[b]{3}{*}{$N$} & \multicolumn{6}{|c|}{ Relative Bias } & \multicolumn{6}{|c|}{ Relative RMSE } \\
\hline & & & \multirow[b]{2}{*}{ ML } & \multirow[b]{2}{*}{ Uninf } & \multicolumn{2}{|c|}{ Corr } & \multicolumn{2}{|c|}{ Missp } & \multirow[b]{2}{*}{ ML } & \multirow[b]{2}{*}{ Uninf } & \multicolumn{2}{|c|}{ Corr } & \multicolumn{2}{|c|}{ Missp } \\
\hline & & & & & $v_{0}=1$ & $v_{0}=3$ & $v_{0}=1$ & $v_{0}=3$ & & & $v_{0}=1$ & $v_{0}=3$ & $v_{0}=1$ & $v_{0}=3$ \\
\hline \multirow{6}{*}{$\hat{\sigma}_{T}^{2}$} & 4 & 200 & -0.30 & -0.04 & 0.00 & -0.02 & 0.07 & 0.09 & 0.61 & 0.42 & 0.22 & 0.19 & 0.22 & 0.21 \\
\hline & 4 & 500 & -0.23 & 0.00 & 0.02 & 0.00 & 0.06 & 0.07 & 0.52 & 0.27 & 0.15 & 0.14 & 0.15 & 0.15 \\
\hline & 6 & 200 & -0.12 & -0.05 & -0.03 & -0.03 & 0.00 & 0.02 & 0.35 & 0.31 & 0.18 & 0.17 & 0.17 & 0.16 \\
\hline & 6 & 500 & -0.05 & -0.01 & -0.01 & -0.02 & 0.00 & 0.01 & 0.20 & 0.17 & 0.13 & 0.11 & 0.13 & 0.11 \\
\hline & 9 & 200 & -0.03 & -0.02 & -0.03 & -0.03 & -0.02 & 0.00 & 0.17 & 0.16 & 0.15 & 0.14 & 0.15 & 0.14 \\
\hline & 9 & 500 & -0.01 & 0.00 & -0.01 & -0.01 & -0.01 & 0.00 & 0.10 & 0.10 & 0.10 & 0.09 & 0.10 & 0.09 \\
\hline \multirow{6}{*}{$\hat{\sigma}_{A}^{2}$} & 4 & 200 & 0.54 & 0.15 & 0.09 & 0.03 & 0.08 & 0.00 & 0.65 & 0.52 & 0.35 & 0.28 & 0.41 & 0.32 \\
\hline & 4 & 500 & 0.41 & 0.15 & 0.10 & 0.05 & 0.13 & 0.06 & 0.54 & 0.29 & 0.25 & 0.20 & 0.27 & 0.23 \\
\hline & 6 & 200 & 0.26 & 0.14 & 0.08 & 0.03 & 0.12 & 0.06 & 0.43 & 0.30 & 0.24 & 0.20 & 0.28 & 0.23 \\
\hline & 6 & 500 & 0.12 & 0.09 & 0.06 & 0.03 & 0.09 & 0.06 & 0.27 & 0.21 & 0.17 & 0.14 & 0.20 & 0.17 \\
\hline & 9 & 200 & 0.10 & 0.07 & 0.05 & 0.02 & 0.07 & 0.05 & 0.27 & 0.22 & 0.19 & 0.15 & 0.23 & 0.19 \\
\hline & 9 & 500 & 0.03 & 0.03 & 0.02 & 0.01 & 0.03 & 0.03 & 0.15 & 0.13 & 0.13 & 0.11 & 0.14 & 0.13 \\
\hline \multirow{5}{*}{$\hat{\sigma}_{S}^{2}$} & 4 & 200 & -0.28 & 0.15 & 0.10 & 0.06 & 0.05 & 0.02 & 0.64 & 0.43 & 0.27 & 0.21 & 0.40 & 0.30 \\
\hline & 4 & 500 & -0.21 & 0.09 & 0.09 & 0.06 & 0.01 & 0.00 & 0.54 & 0.33 & 0.22 & 0.16 & 0.32 & 0.24 \\
\hline & 6 & 200 & -0.17 & 0.02 & 0.05 & 0.05 & -0.03 & -0.01 & 0.50 & 0.40 & 0.25 & 0.18 & 0.36 & 0.27 \\
\hline & 6 & 500 & -0.09 & 0.01 & 0.02 & 0.03 & -0.03 & -0.02 & 0.34 & 0.30 & 0.22 & 0.16 & 0.29 & 0.23 \\
\hline & 9 & 200 & -0.09 & -0.01 & 0.01 & 0.03 & -0.04 & -0.02 & 0.34 & 0.32 & 0.24 & 0.17 & 0.30 & 0.24 \\
\hline \multirow{7}{*}{$\hat{\beta}$} & 9 & 500 & -0.03 & 0.00 & 0.00 & 0.01 & -0.01 & -0.01 & 0.19 & 0.18 & 0.17 & 0.14 & 0.19 & 0.17 \\
\hline & 4 & 200 & 0.03 & 0.15 & 0.00 & 0.01 & -0.27 & -0.31 & 0.57 & 0.60 & 0.30 & 0.13 & 0.47 & 0.43 \\
\hline & 4 & 500 & 0.03 & 0.08 & 0.01 & 0.01 & -0.15 & -0.21 & 0.50 & 0.53 & 0.35 & 0.17 & 0.41 & 0.33 \\
\hline & 6 & 200 & 0.01 & 0.02 & -0.01 & 0.00 & -0.09 & -0.12 & 0.42 & 0.48 & 0.32 & 0.18 & 0.38 & 0.31 \\
\hline & 6 & 500 & 0.00 & -0.02 & -0.02 & 0.00 & -0.07 & -0.08 & 0.30 & 0.35 & 0.27 & 0.19 & 0.32 & 0.27 \\
\hline & 9 & 200 & -0.01 & -0.03 & -0.02 & 0.00 & -0.06 & -0.05 & 0.27 & 0.30 & 0.24 & 0.17 & 0.29 & 0.24 \\
\hline & 9 & 500 & 0.00 & -0.02 & -0.01 & 0.00 & -0.03 & -0.03 & 0.17 & 0.18 & 0.17 & 0.14 & 0.19 & 0.17 \\
\hline
\end{tabular}

Note. $\mathrm{RMSE}=$ root mean squared error. $\mathrm{ML}=$ Maximum Likelihood with constrained estimation. $W$ $=$ number of measurement waves. $N=$ sample size. $v_{0}=$ prior sample size for the prior distributions. Uninf $=$ uninformative prior. Corr $=$ correctly specified (and weakly informative) prior. Missp $=$ misspecified prior. Relative biases smaller than -.10 or larger than .10 are written in bold. 
Constrained maximum likelihood

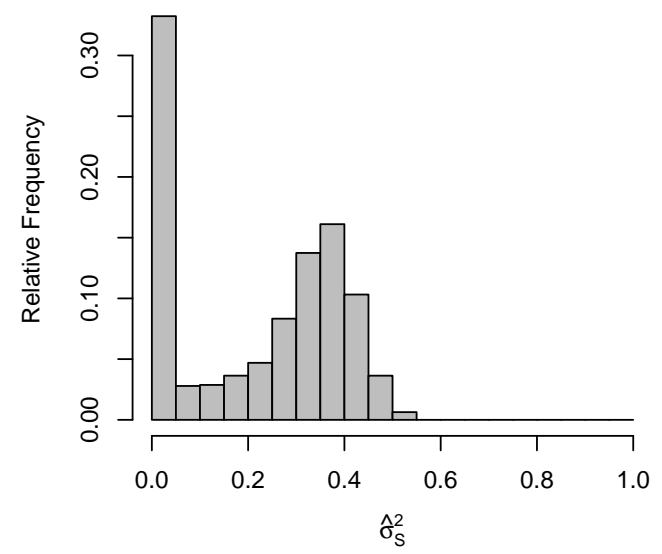

Correctly specified prior

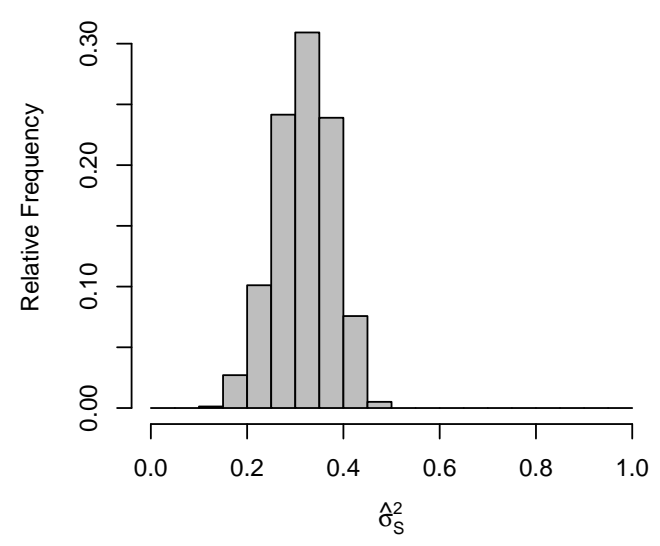

Uninformative prior

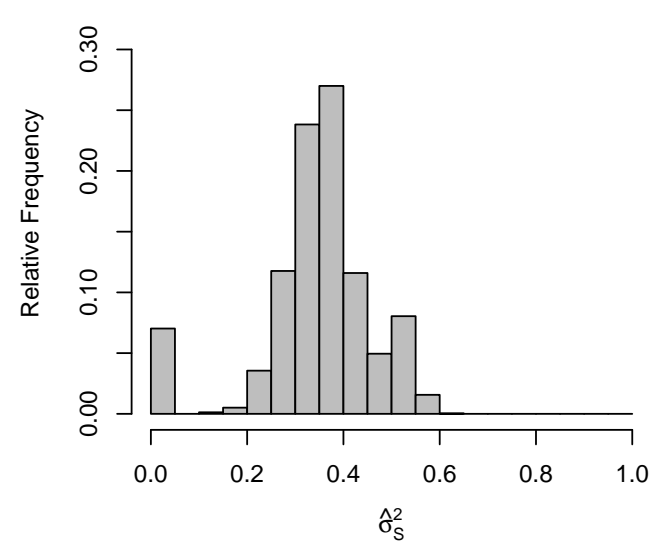

Misspecified prior

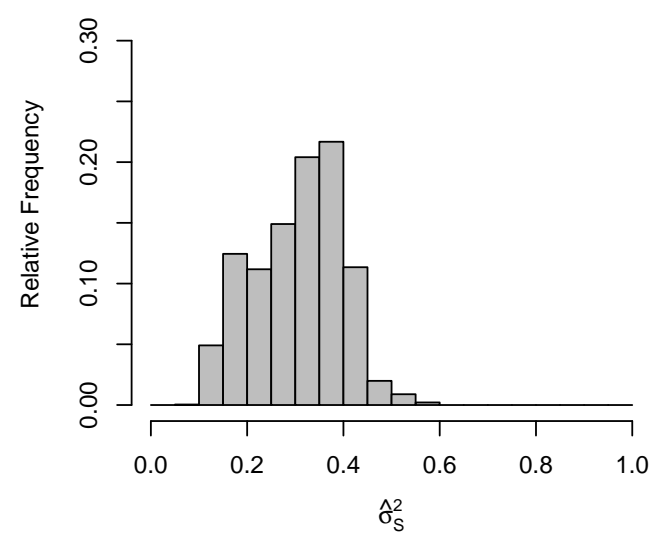

Figure C1. Distribution of parameter estimates $\hat{\theta}_{S}$ for different prior distributions. The ratio of stable trait variance to autoregressive trait variance was 1:1. The state variance was .3. The stability parameter was .5. The number of measurement waves was 4 . The sample size was 200 . Upper left figure: constrained maximum likelihood. Upper right figure: uninformative prior. Lower left figure: correctly specified prior with prior sample size $v_{0}=1$. Lower right figure: misspecified prior with prior sample size $v_{0}=1$. 


\section{Supplement D: Additional Simulation on Highest Posterior Density (HPD) Intervals}

In some of the conditions of the main simulation, the Bayesian approach showed a tendency to produce coverage rates that were too high. In the main simulation, we calculated Bayesian credible intervals (BCI) that were based on quantiles of the posterior distribution. Alternatively, one could calculate highest posterior density (HPD) interval estimates which have a shorter interval length than the BCI.

In an additional small simulation study, we compared the coverage rates produced by the HPD and the BCI for selected conditions of the main simulation with $N=200$ and 500 and $W$ $=4$ and 6 . We held constant the trait variance (.35), the autoregressive trait variance (.35), the state variance (.30), and the stability parameter (.5) of the data-generating model. In the analysis model, we assumed (as in the main simulation) a prior guess of .33 for the stable trait variance, the autoregressive trait variance, and the state factor variance with prior sample sizes of $v_{0}=1$ and $v_{0}=3$.

As can be seen in Table D1, the coverage rates provided by the HPD $(M=98.0 \%$, range $=$ .93 .4 to 100$)$ were indeed slightly smaller than the coverage rates for the BCI $(M=97.6 \%$, range $=93.5$ to 100 ). However, only in three of the 10 cases, in which the coverage rates of the BCI were too high, did the HPD produce appropriate coverage rates, and even in these cases, the differences in the coverage rates between the BCI and HPD were small.

Table D1

Comparison of Coverage Rates for Bayesian Credibility Interval (BCI) and Highest Posterior Density (HPD) Interval Estimates

\begin{tabular}{|c|c|c|c|c|c|c|c|c|}
\hline \multirow[b]{3}{*}{ Parm } & \multicolumn{4}{|c|}{$W=4$} & \multicolumn{4}{|c|}{$W=6$} \\
\hline & \multicolumn{2}{|c|}{$N=200$} & \multicolumn{2}{|c|}{$N=500$} & \multicolumn{2}{|c|}{$N=200$} & \multicolumn{2}{|c|}{$N=500$} \\
\hline & BCI & HPD & BCI & HPD & BCI & HPD & BCI & HPD \\
\hline$\hat{\sigma}_{T}^{2}$ & 98.2 & 97.4 & 98.6 & 98.2 & 96.8 & 96.0 & 95.3 & 95.6 \\
\hline$\hat{\sigma}_{A}^{2}$ & 97.5 & 97.3 & 93.4 & 93.5 & 95.5 & 95.2 & 95.6 & 95.7 \\
\hline$\hat{\sigma}_{S}^{2}$ & 99.7 & 99.6 & 99.9 & 99.6 & 99.9 & 99.3 & 98.7 & 97.2 \\
\hline$\hat{\beta}$ & 100 & 100 & 100 & 100 & 99.8 & 99.7 & 98.7 & 96.8 \\
\hline
\end{tabular}

Note. $W=$ number of measurement waves. $N=$ sample size. Coverage rates smaller than $91 \%$ and larger than $98 \%$ are written in bold. 


\section{Supplement E: Additional Simulation on Small Sample Sizes and Many Measurement Points}

In intensive longitudinal designs or diary studies, a relatively small number of individuals is assessed on many occasions. To further assess the statistical behavior of the Bayesian approach with smaller sample sizes, we conducted a small additional simulation in which we varied the samples sizes $(N=100,200$, and 500) and the number of measurement waves $(W=4,7$, and 14) for a selected condition of the simulation. We held constant the trait variance (.35), the autoregressive trait variance (.35), the state variance (.30), and the stability parameter (.5) of the data-generating model.

Table E1 shows the relative bias and relative RMSE for the estimators provided by the Bayesian approach with a prior sample size of $v_{0}=3$ and prior guesses as specified in the main simulation (.33 for the three variance components and .5 for the stability parameter). Overall, the results showed that a large number of measurement points $(W=14)$ could compensate for a small sample size $(N=100)$, and that the Bayesian approach produced approximately unbiased estimates with an acceptable RMSE under this condition. Thus, we believe that the Bayesian approach offers great potential for analyzing intensive longitudinal data with the STARTS model.

\section{Table E1}

Relative Bias and Relative RMSE for the Bayesian Approach with a Prior Sample Size of $v_{0}=3, a$ Ratio of Stable Trait to Autoregressive Trait Variance of 1:1, a Large State Variance $\left(\sigma_{S}^{2}=.3\right)$ and a Medium Sized Stability Coefficient $(\beta=.5)$

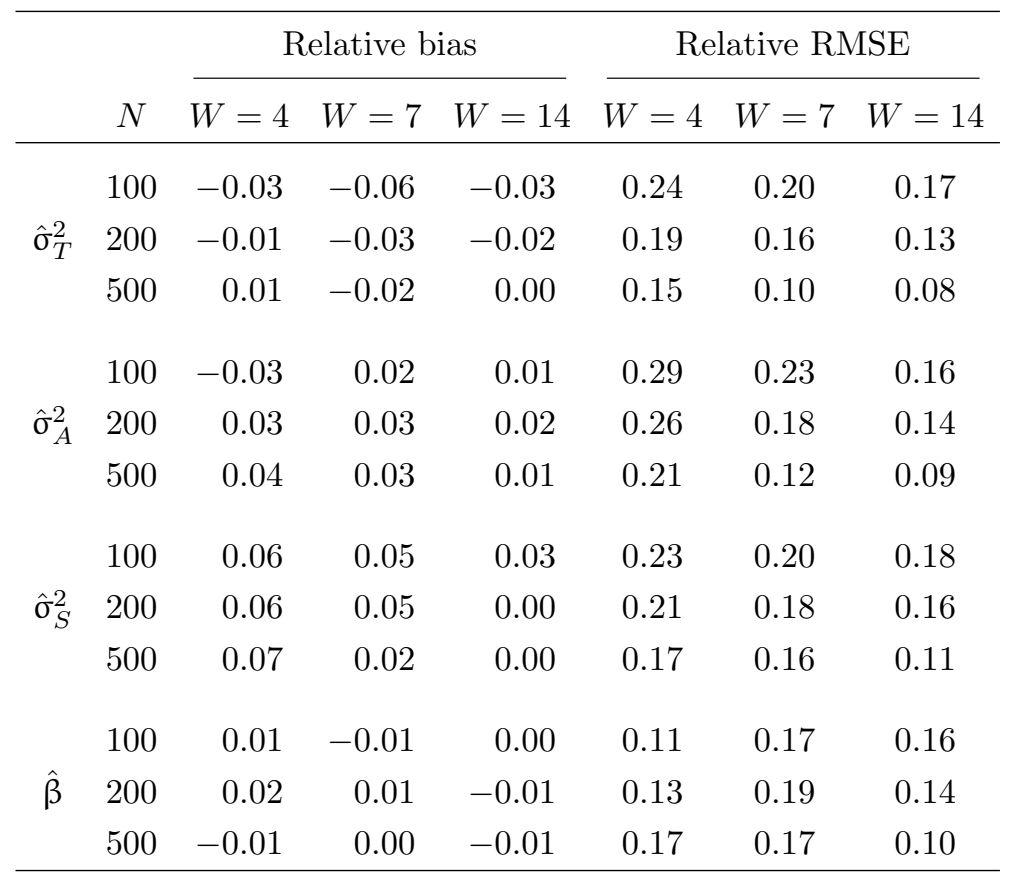

Note. $W=$ number of measurement waves. $N=$ sample size. 


\section{Supplement F: Additional Simulation on More Extreme Conditions}

In the following, we provide results of an additional simulation study in which we looked at more extreme conditions. In this simulation, we set the ratio of the variance of the stable trait factor to the variance of the autoregressive trait factor to $6: 1,1: 6$, and 1:13 (thus, in the most extreme condition the magnitude of the variance of the stable trait was only .05). Again, we specified IG prior distributions with $v_{0}=1,3$, and 10, and a prior guess of .33 for the variance parameters in the analysis models. For the stability parameter, we specified beta distributions with $v_{0}=1,3$, and 10 , and a prior guess of .5. We also varied the sample sizes $(N=200,500$, and 1000) as well as the number of measurement waves ( $W=4$ and 6$)$. In the following, the results are presented in Tables F1 to F6.

The results show that even in these extreme conditions the Bayesian approach still outperformed ML in terms of the RMSE (at least for a prior distribution with $v_{0}=1$ ). It is also evident that the Bayesian estimates have a tendency to be more biased than the ML estimates. However, it needs to be emphasized that in extreme conditions in which the magnitude of the population variance is small (e.g., stable trait variance of .05 in the condition with a ratio of 1:13), the relative bias became very large although the bias was small in absolute terms. For example, in the condition with $N=500$ and $W=6$ (Table F5), a relative bias of .63 for the stable trait variance in the condition with a ratio of $1: 13$ (and a prior sample size of $v_{0}=1$ ) corresponds to an absolute bias of only $.63 \times .05=.03$. In contrast, a relative bias of -.12 for ML estimation of the stable trait variance (in the condition $6: 1$ ) corresponds to an absolute bias of $-.12 \times$ $.60=-.07$. Thus, one needs to be careful to compare the relative biases across very extreme conditions. 
Table F1

Relative Bias, Relative RMSE, and Coverage for the Maximum Likelihood and the Bayesian Approach (with Different Priors) as a Function of the Ratio of Stable Trait to Autoregressive Trait Variance for a Medium Sample Size $(N=200)$, Four Measurement Waves $(W=4)$, a Large State Variance $\left(\sigma_{S}^{2}=.3\right)$ and a Medium Sized Stability Coefficient $(\beta=.5)$

\begin{tabular}{|c|c|c|c|c|c|c|c|c|c|c|c|c|c|}
\hline & \multirow[b]{2}{*}{ Ratio } & \multicolumn{4}{|c|}{ Relative bias } & \multicolumn{4}{|c|}{ Relative RMSE } & \multicolumn{4}{|c|}{ Coverage } \\
\hline & & ML & $v_{0}=1$ & $v_{0}=3$ & $v_{0}=10$ & ML & $v_{0}=1$ & $v_{0}=3$ & $v_{0}=10$ & ML & $v_{0}=1$ & $v_{0}=3$ & $v_{0}=10$ \\
\hline \multirow{3}{*}{$\hat{\boldsymbol{\sigma}}_{T}^{2}$} & $6: 1$ & -0.33 & -0.04 & -0.07 & -0.12 & 0.56 & 0.13 & 0.14 & 0.17 & 97.9 & 95.7 & 93.9 & 87.1 \\
\hline & $1: 6$ & -0.11 & 0.42 & 0.70 & 1.05 & 0.94 & 0.66 & 0.79 & 1.09 & 95.4 & 99.8 & 84.5 & 0.5 \\
\hline & $1: 13$ & 0.29 & 1.37 & 1.99 & 2.77 & 1.54 & 1.60 & 2.08 & 2.81 & 95.6 & 73.2 & 0.0 & 0.0 \\
\hline \multirow{3}{*}{$\hat{\sigma}_{A}^{2}$} & $6: 1$ & 2.78 & 0.45 & 0.70 & 1.01 & 3.63 & 0.74 & 0.82 & 1.05 & 92.8 & 93.8 & 78.3 & 0.4 \\
\hline & $1: 6$ & 0.15 & -0.03 & -0.13 & -0.26 & 0.29 & 0.23 & 0.23 & 0.30 & 99.7 & 98.8 & 96.4 & 77.2 \\
\hline & $1: 13$ & 0.12 & -0.05 & -0.17 & -0.30 & 0.27 & 0.22 & 0.25 & 0.33 & 99.8 & 98.9 & 94.2 & 61.6 \\
\hline \multirow{3}{*}{$\hat{\sigma}_{S}^{2}$} & $6: 1$ & -0.28 & -0.03 & -0.10 & -0.17 & 0.57 & 0.11 & 0.13 & 0.18 & 88.0 & 98.2 & 94.5 & 73.7 \\
\hline & $1: 6$ & -0.27 & -0.08 & 0.06 & 0.21 & 0.66 & 0.42 & 0.31 & 0.29 & 96.7 & 99.7 & 100 & 99.2 \\
\hline & $1: 13$ & -0.31 & -0.14 & 0.05 & 0.25 & 0.68 & 0.46 & 0.33 & 0.33 & 97.3 & 99.7 & 100 & 98.9 \\
\hline \multirow{3}{*}{$\hat{\beta}$} & $6: 1$ & 0.25 & -0.12 & -0.06 & 0.04 & 0.82 & 0.53 & 0.23 & 0.08 & 74.3 & 100 & 100 & 100 \\
\hline & $1: 6$ & -0.06 & -0.17 & -0.15 & -0.10 & 0.37 & 0.22 & 0.17 & 0.11 & 99.6 & 100 & 100 & 100 \\
\hline & $1: 13$ & -0.10 & -0.22 & -0.19 & -0.13 & 0.33 & 0.25 & 0.20 & 0.14 & 99.8 & 99.8 & 100 & 100 \\
\hline
\end{tabular}

Note. RMSE $=$ root mean square error. $\mathrm{ML}=$ Maximum Likelihood with constrained estimation. $v_{0}$ $=$ prior sample size selected the for the prior distributions in the Bayesian approach. Ratio $=$ ratio of stable trait variance to autoregressive trait variance. Relative biases smaller than -.10 or larger than .10 and coverage rates smaller than $91 \%$ and larger than $98 \%$ are written in bold. 
Table F2

Relative Bias, Relative RMSE, and Coverage for the Maximum Likelihood and the Bayesian Approach (with Different Priors) as a Function of the Ratio of Stable Trait to Autoregressive Trait Variance for a Medium Sample Size $(N=500)$, Four Measurement Waves $(W=4)$, a Large State Variance $\left(\sigma_{S}^{2}=.3\right)$ and a Medium Sized Stability Coefficient $(\beta=.5)$

\begin{tabular}{|c|c|c|c|c|c|c|c|c|c|c|c|c|c|}
\hline & \multirow[b]{2}{*}{ Ratio } & \multicolumn{4}{|c|}{ Relative bias } & \multicolumn{4}{|c|}{ Relative RMSE } & \multicolumn{4}{|c|}{ Coverage } \\
\hline & & ML & $v_{0}=1$ & $v_{0}=3$ & $v_{0}=10$ & ML & $v_{0}=1$ & $v_{0}=3$ & $v_{0}=10$ & ML & $v_{0}=1$ & $v_{0}=3$ & $v_{0}=10$ \\
\hline \multirow{3}{*}{$\hat{\boldsymbol{\sigma}}_{T}^{2}$} & $6: 1$ & -0.30 & -0.01 & -0.03 & -0.06 & 0.53 & 0.08 & 0.08 & 0.10 & 97.8 & 97.0 & 96.0 & 91.4 \\
\hline & $1: 6$ & -0.10 & 0.34 & 0.53 & 0.81 & 0.81 & 0.52 & 0.61 & 0.84 & 94.4 & 99.6 & 91.7 & 2.8 \\
\hline & $1: 13$ & 0.15 & 1.06 & 1.54 & 2.19 & 1.26 & 1.21 & 1.61 & 2.22 & 95.8 & 83.4 & 0.4 & 0.0 \\
\hline \multirow{3}{*}{$\hat{\sigma}_{A}^{2}$} & $6: 1$ & 2.66 & 0.29 & 0.48 & 0.75 & 3.42 & 0.50 & 0.57 & 0.78 & 99.0 & 94.5 & 84.0 & 7.1 \\
\hline & $1: 6$ & 0.12 & 0.03 & -0.04 & -0.15 & 0.23 & 0.16 & 0.14 & 0.19 & 99.3 & 98.6 & 98.8 & 88.0 \\
\hline & $1: 13$ & 0.09 & 0.02 & -0.06 & -0.18 & 0.21 & 0.15 & 0.14 & 0.21 & 99.2 & 98.6 & 98.2 & 79.2 \\
\hline \multirow{3}{*}{$\hat{\sigma}_{S}^{2}$} & $6: 1$ & -0.29 & 0.02 & -0.04 & -0.12 & 0.56 & 0.08 & 0.08 & 0.13 & 86.5 & 99.5 & 98.0 & 78.1 \\
\hline & $1: 6$ & -0.21 & -0.13 & -0.06 & 0.07 & 0.56 & 0.34 & 0.24 & 0.18 & 97.9 & 98.9 & 99.8 & 100 \\
\hline & $1: 13$ & -0.23 & -0.24 & -0.12 & 0.07 & 0.55 & 0.40 & 0.27 & 0.19 & 98.6 & 97.7 & 99.4 & 99.8 \\
\hline \multirow{3}{*}{$\hat{\beta}$} & $6: 1$ & 0.14 & -0.11 & -0.11 & 0.00 & 0.79 & 0.59 & 0.32 & 0.09 & 63.5 & 100 & 100 & 100 \\
\hline & $1: 6$ & -0.05 & -0.16 & -0.15 & -0.13 & 0.32 & 0.21 & 0.17 & 0.14 & 99.3 & 99.5 & 99.6 & 99.8 \\
\hline & $1: 13$ & -0.08 & -0.22 & -0.21 & -0.17 & 0.27 & 0.24 & 0.22 & 0.18 & 99.6 & 96.2 & 96.0 & 97.3 \\
\hline
\end{tabular}

Note. RMSE $=$ root mean square error. $\mathrm{ML}=$ Maximum Likelihood with constrained estimation. $v_{0}$ $=$ prior sample size selected the for the prior distributions in the Bayesian approach. Ratio $=$ ratio of stable trait variance to autoregressive trait variance. Relative biases smaller than -.10 or larger than .10 and coverage rates smaller than $91 \%$ and larger than $98 \%$ are written in bold. 
Table F3

Relative Bias, Relative RMSE, and Coverage for the Maximum Likelihood and the Bayesian Approach (with Different Priors) as a Function of the Ratio of Stable Trait to Autoregressive Trait Variance for a Large Sample Size $(N=1000)$, Four Measurement Waves $(W=4)$, a Large State Variance $\left(\sigma_{S}^{2}=.3\right)$ and a Medium Sized Stability Coefficient $(\beta=.5)$

\begin{tabular}{|c|c|c|c|c|c|c|c|c|c|c|c|c|c|}
\hline & \multirow[b]{2}{*}{ Ratio } & \multicolumn{4}{|c|}{ Relative bias } & \multicolumn{4}{|c|}{ Relative RMSE } & \multicolumn{4}{|c|}{ Coverage } \\
\hline & & ML & $v_{0}=1$ & $v_{0}=3$ & $v_{0}=10$ & ML & $v_{0}=1$ & $v_{0}=3$ & $v_{0}=10$ & ML & $v_{0}=1$ & $v_{0}=3$ & $v_{0}=10$ \\
\hline \multirow{3}{*}{$\hat{\boldsymbol{\sigma}}_{T}^{2}$} & $6: 1$ & -0.25 & 0.00 & -0.01 & -0.04 & 0.48 & 0.06 & 0.06 & 0.07 & 97.6 & 97.6 & 97.0 & 93.7 \\
\hline & $1: 6$ & -0.09 & 0.31 & 0.43 & 0.65 & 0.72 & 0.46 & 0.50 & 0.67 & 93.3 & 99.4 & 94.5 & 9.4 \\
\hline & $1: 13$ & 0.13 & 0.95 & 1.30 & 1.83 & 1.16 & 1.08 & 1.36 & 1.85 & 93.1 & 86.3 & 1.2 & 0.0 \\
\hline \multirow{3}{*}{$\hat{\sigma}_{A}^{2}$} & $6: 1$ & 2.30 & 0.22 & 0.35 & 0.57 & 3.10 & 0.39 & 0.43 & 0.60 & 99.9 & 93.6 & 85.7 & 21.5 \\
\hline & $1: 6$ & 0.09 & 0.04 & 0.00 & -0.08 & 0.18 & 0.13 & 0.11 & 0.12 & 99.0 & 96.8 & 98.7 & 94.7 \\
\hline & $1: 13$ & 0.08 & 0.05 & -0.01 & -0.10 & 0.17 & 0.13 & 0.10 & 0.13 & 99.4 & 97.7 & 99.2 & 92.2 \\
\hline \multirow{3}{*}{$\hat{\sigma}_{S}^{2}$} & $6: 1$ & -0.26 & 0.03 & -0.02 & -0.09 & 0.52 & 0.08 & 0.06 & 0.10 & 86.4 & 99.7 & 98.9 & 86.1 \\
\hline & $1: 6$ & -0.15 & -0.13 & -0.11 & -0.02 & 0.47 & 0.30 & 0.22 & 0.14 & 97.9 & 97.9 & 98.8 & 99.8 \\
\hline & $1: 13$ & -0.19 & -0.26 & -0.19 & -0.06 & 0.48 & 0.38 & 0.27 & 0.15 & 98.8 & 94.2 & 96.7 & 99.9 \\
\hline \multirow{3}{*}{$\hat{\beta}$} & $6: 1$ & 0.10 & -0.09 & -0.11 & -0.04 & 0.74 & 0.60 & 0.37 & 0.13 & 64.1 & 100 & 100 & 100 \\
\hline & $1: 6$ & -0.03 & -0.14 & -0.15 & -0.14 & 0.27 & 0.20 & 0.17 & 0.15 & 98.4 & 99.3 & 98.8 & 98.2 \\
\hline & $1: 13$ & -0.07 & -0.20 & -0.21 & -0.19 & 0.25 & 0.23 & 0.22 & 0.19 & 98.8 & 90.3 & 83.0 & 74.1 \\
\hline
\end{tabular}

Note. RMSE $=$ root mean square error. $\mathrm{ML}=$ Maximum Likelihood with constrained estimation. $v_{0}$ $=$ prior sample size selected the for the prior distributions in the Bayesian approach. Ratio $=$ ratio of stable trait variance to autoregressive trait variance. Relative biases smaller than -.10 or larger than .10 and coverage rates smaller than $91 \%$ and larger than $98 \%$ are written in bold. 
Table F4

Relative Bias, Relative RMSE, and Coverage for the Maximum Likelihood and the Bayesian Approach (with Different Priors) as a Function of the Ratio of Stable Trait to Autoregressive Trait Variance for a Medium Sample Size $(N=200)$, Six Measurement Waves $(W=6)$, a Large State Variance $\left(\sigma_{S}^{2}=.3\right)$ and a Medium Sized Stability Coefficient $(\beta=.5)$

\begin{tabular}{|c|c|c|c|c|c|c|c|c|c|c|c|c|c|}
\hline & \multirow[b]{2}{*}{ Ratio } & \multicolumn{4}{|c|}{ Relative bias } & \multicolumn{4}{|c|}{ Relative RMSE } & \multicolumn{4}{|c|}{ Coverage } \\
\hline & & ML & $v_{0}=1$ & $v_{0}=3$ & $v_{0}=10$ & ML & $v_{0}=1$ & $v_{0}=3$ & $v_{0}=10$ & ML & $v_{0}=1$ & $v_{0}=3$ & $v_{0}=10$ \\
\hline \multirow{3}{*}{$\hat{\boldsymbol{\sigma}}_{T}^{2}$} & $6: 1$ & -0.23 & -0.03 & -0.04 & -0.07 & 0.48 & 0.12 & 0.12 & 0.13 & 97.4 & 95.8 & 95.4 & 92.7 \\
\hline & $1: 6$ & -0.13 & 0.20 & 0.42 & 0.76 & 0.68 & 0.42 & 0.51 & 0.79 & 97.2 & 99.3 & 92.3 & 6.6 \\
\hline & $1: 13$ & 0.02 & 0.82 & 1.34 & 2.09 & 1.03 & 0.98 & 1.41 & 2.13 & 96.6 & 87.1 & 1.3 & 0.0 \\
\hline \multirow{3}{*}{$\hat{\sigma}_{A}^{2}$} & $6: 1$ & 2.10 & 0.24 & 0.41 & 0.68 & 3.01 & 0.44 & 0.50 & 0.71 & 98.9 & 94.9 & 86.6 & 10.9 \\
\hline & $1: 6$ & 0.08 & 0.02 & -0.04 & -0.14 & 0.22 & 0.17 & 0.15 & 0.18 & 98.8 & 98.3 & 98.5 & 91.4 \\
\hline & $1: 13$ & 0.07 & 0.03 & -0.05 & -0.16 & 0.21 & 0.16 & 0.14 & 0.19 & 98.9 & 98.6 & 99.2 & 86.9 \\
\hline \multirow{3}{*}{$\hat{\sigma}_{S}^{2}$} & $6: 1$ & -0.23 & 0.00 & -0.06 & -0.14 & 0.50 & 0.10 & 0.10 & 0.15 & 90.2 & 99.2 & 96.8 & 78.1 \\
\hline & $1: 6$ & -0.12 & -0.09 & -0.02 & 0.09 & 0.50 & 0.34 & 0.24 & 0.19 & 95.4 & 99.3 & 99.7 & 99.7 \\
\hline & $1: 13$ & -0.17 & -0.20 & -0.09 & 0.07 & 0.51 & 0.39 & 0.26 & 0.19 & 97.2 & 98.7 & 99.7 & 99.9 \\
\hline \multirow{3}{*}{$\hat{\beta}$} & $6: 1$ & 0.10 & -0.18 & -0.19 & -0.08 & 0.69 & 0.55 & 0.34 & 0.14 & 68.6 & 100 & 100 & 100 \\
\hline & $1: 6$ & 0.00 & -0.11 & -0.11 & -0.08 & 0.28 & 0.19 & 0.15 & 0.10 & 98.9 & 99.8 & 99.8 & 100 \\
\hline & $1: 13$ & -0.04 & -0.17 & -0.16 & -0.12 & 0.24 & 0.21 & 0.18 & 0.13 & 99.0 & 98.3 & 98.7 & 99.9 \\
\hline
\end{tabular}

Note. RMSE $=$ root mean square error. $\mathrm{ML}=$ Maximum Likelihood with constrained estimation. $v_{0}$ $=$ prior sample size selected the for the prior distributions in the Bayesian approach. Ratio $=$ ratio of stable trait variance to autoregressive trait variance. Relative biases smaller than -.10 or larger than .10 and coverage rates smaller than $91 \%$ and larger than $98 \%$ are written in bold. 
Table F5

Relative Bias, Relative RMSE, and Coverage for the Maximum Likelihood and the Bayesian Approach (with Different Priors) as a Function of the Ratio of Stable Trait to Autoregressive Trait Variance for a Medium Sample Size $(N=500)$, Six Measurement Waves $(W=6)$, a Large State Variance $\left(\sigma_{S}^{2}=.3\right)$ and a Medium Sized Stability Coefficient $(\beta=.5)$

\begin{tabular}{|c|c|c|c|c|c|c|c|c|c|c|c|c|c|}
\hline & \multirow[b]{2}{*}{ Ratio } & \multicolumn{4}{|c|}{ Relative bias } & \multicolumn{4}{|c|}{ Relative RMSE } & \multicolumn{4}{|c|}{ Coverage } \\
\hline & & ML & $v_{0}=1$ & $v_{0}=3$ & $v_{0}=10$ & ML & $v_{0}=1$ & $v_{0}=3$ & $v_{0}=10$ & ML & $v_{0}=1$ & $v_{0}=3$ & $v_{0}=10$ \\
\hline \multirow{3}{*}{$\hat{\boldsymbol{\sigma}}_{T}^{2}$} & $6: 1$ & -0.12 & -0.01 & -0.02 & -0.03 & 0.33 & 0.08 & 0.08 & 0.08 & 97.0 & 96.2 & 95.3 & 94.4 \\
\hline & $1: 6$ & -0.09 & 0.13 & 0.28 & 0.52 & 0.52 & 0.32 & 0.36 & 0.55 & 96.6 & 98.6 & 95.3 & 26.4 \\
\hline & $1: 13$ & 0.01 & 0.63 & 1.01 & 1.57 & 0.83 & 0.77 & 1.06 & 1.59 & 95.9 & 88.8 & 10.2 & 0.0 \\
\hline \multirow{3}{*}{$\hat{\sigma}_{A}^{2}$} & $6: 1$ & 1.28 & 0.16 & 0.27 & 0.47 & 2.18 & 0.31 & 0.34 & 0.50 & 99.3 & 94.2 & 88.0 & 32.6 \\
\hline & $1: 6$ & 0.05 & 0.03 & 0.00 & -0.05 & 0.16 & 0.13 & 0.11 & 0.10 & 98.1 & 96.1 & 98.5 & 97.3 \\
\hline & $1: 13$ & 0.05 & 0.04 & 0.00 & -0.07 & 0.15 & 0.13 & 0.10 & 0.11 & 97.8 & 96.8 & 99.0 & 96.3 \\
\hline \multirow{3}{*}{$\hat{\sigma}_{S}^{2}$} & $6: 1$ & -0.18 & 0.01 & -0.04 & -0.10 & 0.42 & 0.10 & 0.08 & 0.12 & 89.4 & 99.1 & 97.4 & 83.5 \\
\hline & $1: 6$ & -0.08 & -0.09 & -0.08 & -0.02 & 0.37 & 0.28 & 0.21 & 0.14 & 94.9 & 97.7 & 98.7 & 99.7 \\
\hline & $1: 13$ & -0.10 & -0.18 & -0.15 & -0.06 & 0.37 & 0.33 & 0.25 & 0.15 & 97.5 & 94.5 & 97.0 & 99.7 \\
\hline \multirow{3}{*}{$\hat{\beta}$} & $6: 1$ & 0.02 & -0.15 & -0.19 & -0.16 & 0.58 & 0.53 & 0.37 & 0.21 & 78.9 & 99.9 & 100 & 100 \\
\hline & $1: 6$ & 0.00 & -0.08 & -0.09 & -0.10 & 0.21 & 0.16 & 0.14 & 0.11 & 96.6 & 98.6 & 98.4 & 98.7 \\
\hline & $1: 13$ & -0.03 & -0.14 & -0.15 & -0.14 & 0.19 & 0.18 & 0.17 & 0.15 & 97.4 & 92.1 & 88.9 & 87.5 \\
\hline
\end{tabular}

Note. RMSE $=$ root mean square error. $\mathrm{ML}=$ Maximum Likelihood with constrained estimation. $v_{0}$ $=$ prior sample size selected the for the prior distributions in the Bayesian approach. Ratio $=$ ratio of stable trait variance to autoregressive trait variance. Relative biases smaller than -.10 or larger than .10 and coverage rates smaller than $91 \%$ and larger than $98 \%$ are written in bold. 
Table F6

Relative Bias, Relative RMSE, and Coverage for the Maximum Likelihood and the Bayesian Approach (with Different Priors) as a Function of the Ratio of Stable Trait to Autoregressive Trait Variance for a Large Sample Size $(N=1000)$, Six Measurement Waves $(W=6)$, a Large State Variance $\left(\sigma_{S}^{2}=.3\right)$ and a Medium Sized Stability Coefficient $(\beta=.5)$

\begin{tabular}{|c|c|c|c|c|c|c|c|c|c|c|c|c|c|}
\hline & \multirow[b]{2}{*}{ Ratio } & \multicolumn{4}{|c|}{ Relative bias } & \multicolumn{4}{|c|}{ Relative RMSE } & \multicolumn{4}{|c|}{ Coverage } \\
\hline & & ML & $v_{0}=1$ & $v_{0}=3$ & $v_{0}=10$ & ML & $v_{0}=1$ & $v_{0}=3$ & $v_{0}=10$ & ML & $v_{0}=1$ & $v_{0}=3$ & $v_{0}=10$ \\
\hline \multirow{3}{*}{$\hat{\sigma}_{T}^{2}$} & $6: 1$ & -0.06 & -0.01 & -0.01 & -0.01 & 0.22 & 0.06 & 0.06 & 0.05 & 97.2 & 96.0 & 95.7 & 94.4 \\
\hline & $1: 6$ & -0.05 & 0.10 & 0.21 & 0.39 & 0.39 & 0.27 & 0.28 & 0.41 & 96.1 & 98.0 & 94.8 & 46.0 \\
\hline & $1: 13$ & -0.04 & 0.48 & 0.78 & 1.23 & 0.65 & 0.59 & 0.82 & 1.25 & 96.5 & 91.0 & 22.9 & 0.0 \\
\hline \multirow{3}{*}{$\hat{\sigma}_{A}^{2}$} & $6: 1$ & 0.75 & 0.13 & 0.20 & 0.35 & 1.50 & 0.27 & 0.27 & 0.37 & 99.3 & 92.9 & 88.2 & 48.1 \\
\hline & 1:6 & 0.03 & 0.02 & 0.01 & -0.02 & 0.10 & 0.10 & 0.08 & 0.07 & 97.2 & 95.7 & 96.9 & 98.5 \\
\hline & $1: 13$ & 0.02 & 0.03 & 0.02 & -0.02 & 0.10 & 0.10 & 0.08 & 0.07 & 97.5 & 95.2 & 97.5 & 98.5 \\
\hline \multirow{3}{*}{$\hat{\sigma}_{S}^{2}$} & $6: 1$ & -0.13 & 0.00 & -0.03 & -0.09 & 0.34 & 0.10 & 0.08 & 0.10 & 91.7 & 97.4 & 96.3 & 85.1 \\
\hline & 1:6 & -0.03 & -0.05 & -0.07 & -0.06 & 0.26 & 0.22 & 0.18 & 0.13 & 94.7 & 96.5 & 97.1 & 98.8 \\
\hline & $1: 13$ & -0.05 & -0.14 & -0.16 & -0.12 & 0.25 & 0.25 & 0.23 & 0.17 & 97.2 & 93.2 & 92.7 & 97.0 \\
\hline \multirow{3}{*}{$\hat{\beta}$} & $6: 1$ & -0.01 & -0.11 & -0.16 & -0.19 & 0.49 & 0.48 & 0.37 & 0.25 & 87.0 & 98.4 & 99.0 & 98.9 \\
\hline & $1: 6$ & 0.00 & -0.05 & -0.07 & -0.09 & 0.16 & 0.14 & 0.12 & 0.11 & 95.2 & 97.2 & 96.8 & 94.8 \\
\hline & $1: 13$ & -0.01 & -0.11 & -0.14 & -0.15 & 0.14 & 0.15 & 0.15 & 0.16 & 97.4 & 91.0 & 81.4 & 63.0 \\
\hline
\end{tabular}

Note. RMSE $=$ root mean square error. $\mathrm{ML}=$ Maximum Likelihood with constrained estimation. $v_{0}$ $=$ prior sample size selected the for the prior distributions in the Bayesian approach. Ratio = ratio of stable trait variance to autoregressive trait variance. Relative biases smaller than -.10 or larger than .10 and coverage rates smaller than $91 \%$ and larger than $98 \%$ are written in bold. 


\section{Supplement G: Software Code for the Data Analysis Example}

In the following, we present $\mathrm{R}$ code for estimating the STARTS model with five measurement waves for the data analysis example (Trautwein et al., 2010) in the Mplus software as well in the lavaan and the STARTS $R$ packages.

\section{G.1 lavaan Code}

Below is the $\mathrm{R}$ code for estimating the STARTS model with five measurement waves with the lavaan package. The estimation method is constrained maximum likelihood.

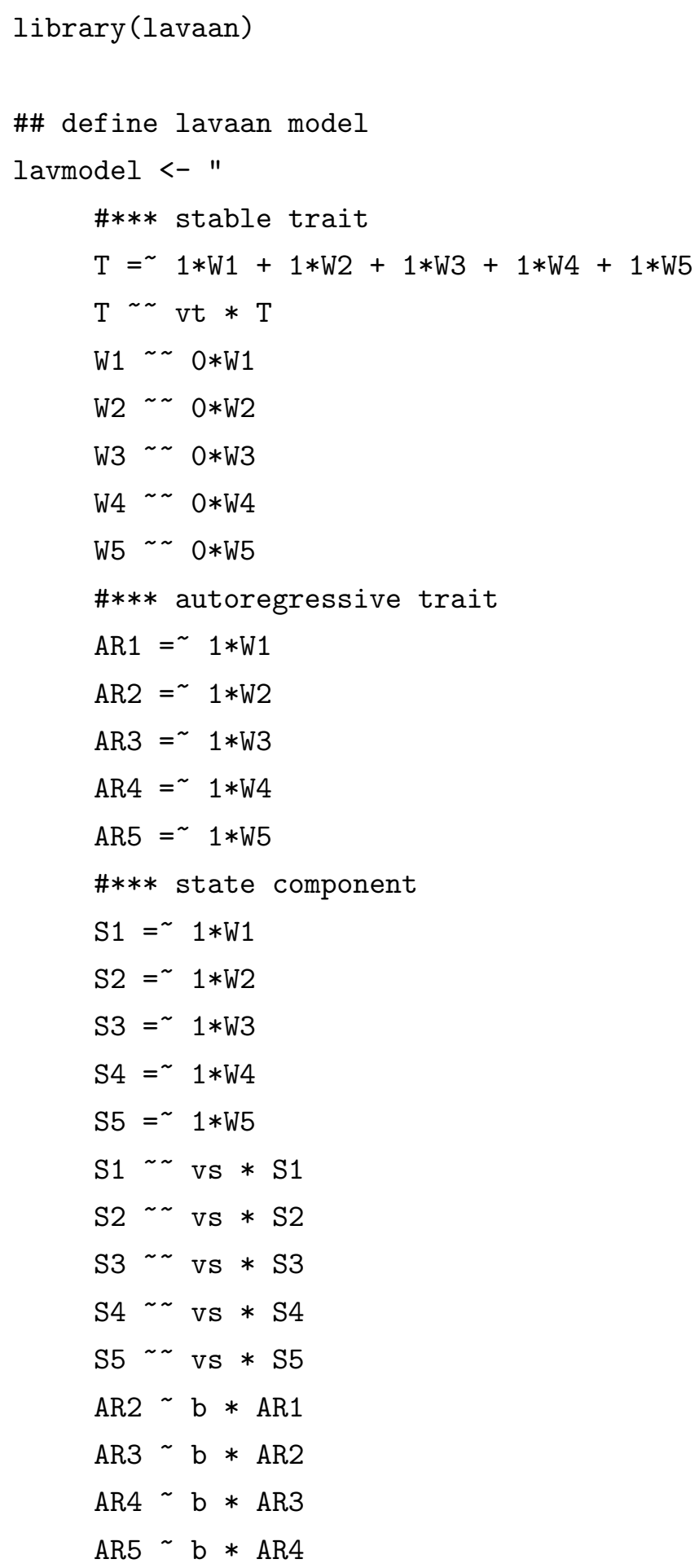




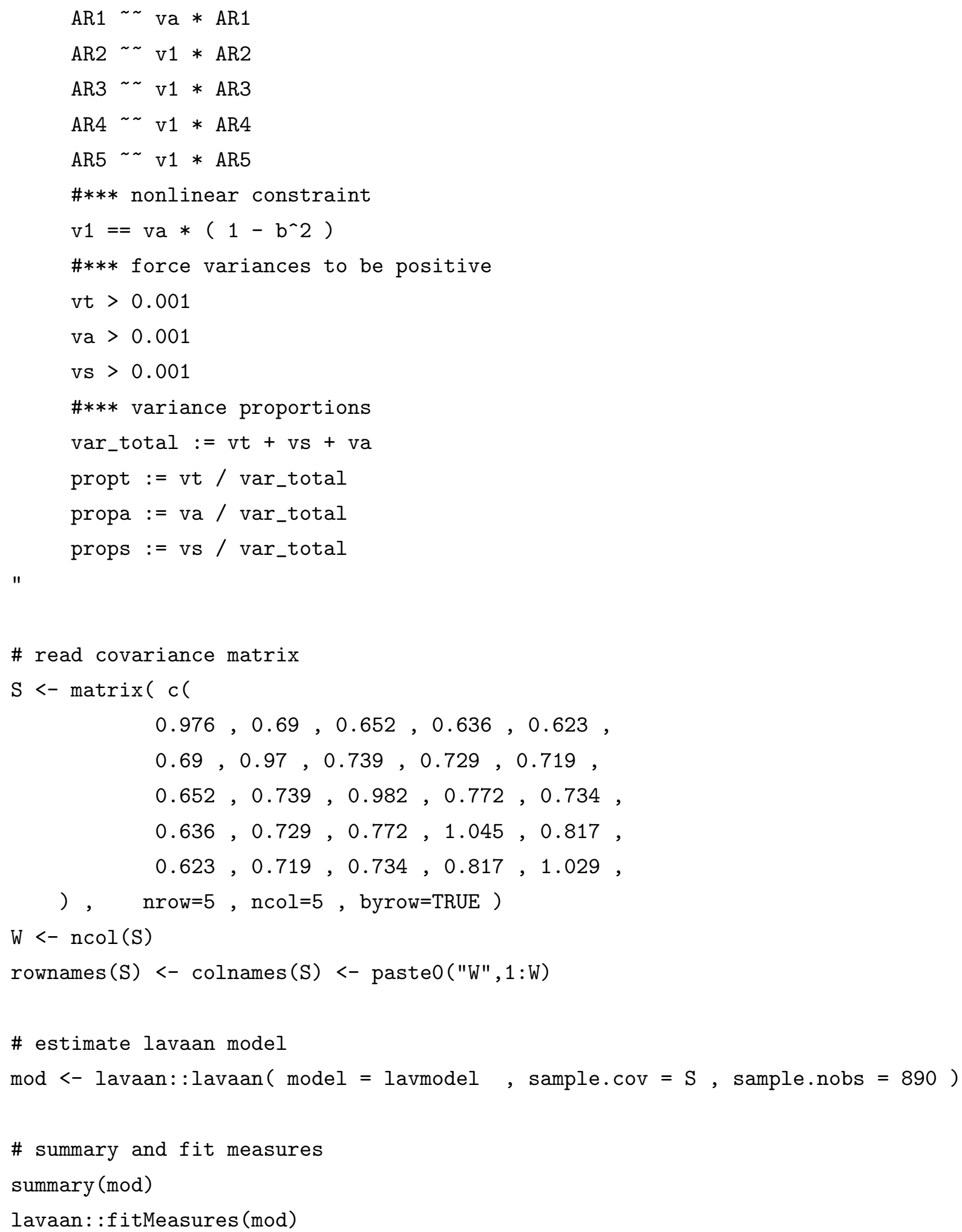

\section{G.2 Mplus Code}

Below is Mplus syntax for estimating the STARTS model with five measurement waves. The estimation method is constrained maximum likelihood. 
DATA :

FILE is covdata.dat ;

TYPE IS COVARIANCE;

NOBSERVATIONS $=890$;

VARIABLE:

NAMES ARE

W1 W2 W3 W4 W5;

USEVARIABLES ARE

W1 W2 W3 W4 W5;

ANALYSIS :

Model $=$ NOCOVARIANCES;

MODEL :

!*** stable trait;

$\mathrm{T}$ by W1-W5@1;

$\mathrm{T}$ (vt);

!*** autoregressive trait

ar1 by W1@1;

ar2 by W2@1;

ar3 by W301;

ar4 by W4@1;

ar5 by W5@1;

N1-N5@0;

!*** state component;

s1 by W1@1;

s2 by W2@1;

s3 by W301;

s4 by W4@1;

s5 by W501;

s1-s5 (vs);

!*** autoregressive process

ar2 on $\operatorname{ar} 1 * .8$ (b);

ar3 on $\operatorname{ar} 2 * .8$ (b);

ar4 on $\operatorname{ar} 3 * .8$ (b);

ar5 on $\operatorname{ar} 4 * .8$ (b);

ar1 (va);

ar2 (v1);

$\operatorname{ar3}$ (v1);

ar4 (v1); 
$\operatorname{ar5}(\mathrm{v} 1)$;

MODEL CONSTRAINT :

NEW (propt, propa, props, vartotal);

!*** nonlinear constraint

$\mathrm{v} 1=\mathrm{va} *(1-\mathrm{b} * \mathrm{~b}) ;$

!*** inequality constraint

vt >0.001;

va $>0.001$

vs >0.001;

!*** inference for variance proportions

vartotal $=\mathrm{vt}+\mathrm{vs}+\mathrm{va} ;$

propt = vt / vartotal;

propa = va / vartotal;

props = vs / vartotal;

OUTPUT :

TECH4; CINTERVAL;

\section{G.3 STARTS and LAM Code}

Below is code for estimating the STARTS model with prior distributions using the STARTS and LAM packages and the LAM: : amh function.

\#\# attach needed packages

library (STARTS)

library (LAM)

\#\# define list with input data

\#\# S ... covariance matrix, M ... mean vector

$\mathrm{S}<-\operatorname{matrix}(\mathrm{c}($

$$
\begin{aligned}
& 0.976,0.69,0.652,0.636,0.623, \\
& 0.69,0.97,0.739,0.729,0.719, \\
& 0.652,0.739,0.982,0.772,0.734, \\
& 0.636,0.729,0.772,1.045,0.817, \\
& 0.623,0.719,0.734,0.817,1.029, \\
& \text { nrow=5 }, \text { ncol=5, byrow=TRUE ) }
\end{aligned}
$$

$\mathrm{W}<-\mathrm{ncol}(\mathrm{S}) \quad \#$ number of measurement waves

data <- list ( $\mathrm{S} "=\mathrm{S}, " \mathrm{M} "=\operatorname{rep}(0, \mathrm{~W}), " \mathrm{n} "=890, " \mathrm{~W} "=\mathrm{W}$ )

\#*** likelihood function for the STARTS model

model <- function( pars, data ) \{ 


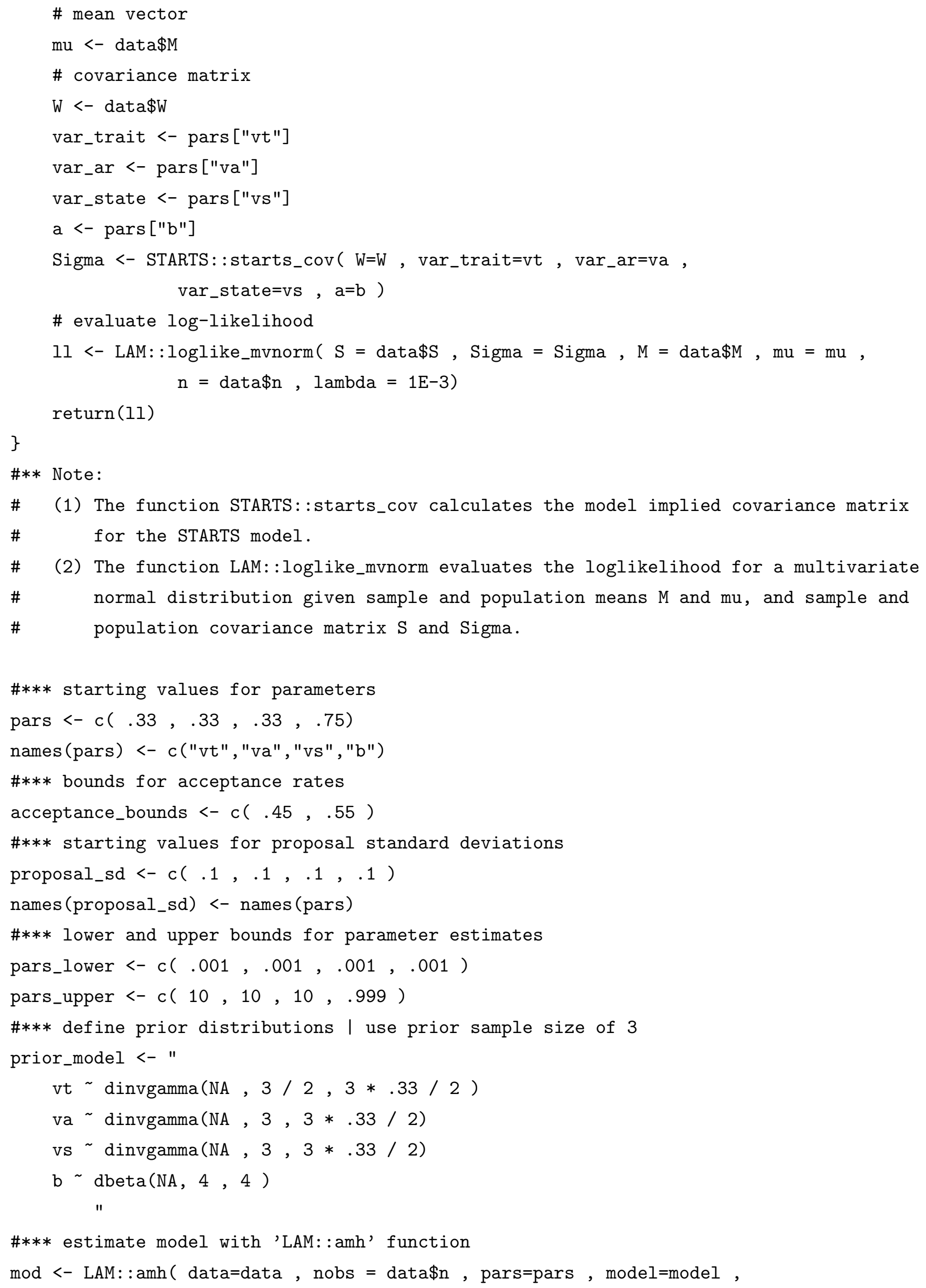




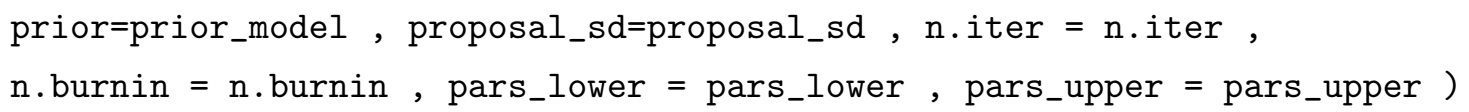

